\title{
Does $C^{*}$-embedding imply $C$-embedding in the realm of products with a non-discrete metric factor?
}

\author{
by
}

\author{
Valentin G u tev (Durban) and Haruto O h t a (Shizuoka)
}

\begin{abstract}
The above question was raised by Teodor Przymusiński in May, 1983, in an unpublished manuscript of his. Later on, it was recognized by Takao Hoshina as a question that is of fundamental importance in the theory of rectangular normality. The present paper provides a complete affirmative solution. The technique developed for the purpose allows one to answer also another question of Przymusiński's.
\end{abstract}

1. Introduction. A subset $A$ of a topological space $X$ is $C^{*}$-embedded in $X$ if every bounded real-valued continuous function on $A$ is continuously extendable to the whole of $X$. If this holds for all real-valued continuous functions on $A$, then $A$ is called $C$-embedded in $X$. Now, we shall say that a subset $A \subset X$ is $U^{\omega}$-embedded in $X$ if for every continuous function $f$ : $A \rightarrow \mathbb{R}$, there exists a continuous function $g: X \rightarrow \mathbb{R}$ such that $f(x) \leq g(x)$ whenever $x \in A$. The notion " $U^{\omega}$-embedded" in this sense is the same as " $U^{\omega}$-embedded" in the sense of Hoshina [8] (see Lemma 2.5). It should be mentioned that a subset $A \subset X$ is $C$-embedded in $X$ if and only if it is both $U^{\omega}$ - and $C^{*}$-embedded in $X[20]$ (see [8, Proposition 1.6]).

Another special embedding which will play a central role in the paper is related to extension of maps with values in Banach spaces. Let $\lambda$ be an infinite cardinal. A subset $A \subset X$ is $P^{\lambda}$-embedded in $X$ if every continuous map $f: A \rightarrow Y$ into a Banach space $Y$ with $w(Y) \leq \lambda$ is continuously extendable over $X$, where $w(Y)$ is the weight of $Y$, i.e., the least cardinality of a base for the topology of $Y$. The notion " $P^{\lambda}$-embedded" in this sense is the same as " $P^{\lambda}$-embedded" in the sense of Shapiro [28], which was introduced by Arens [3] under the name " $\lambda$-normally embedded" (see $[1,19,22$, $28])$. It should be mentioned that $A$ is $C$-embedded in $X$ if and only if it is $P^{\omega}$-embedded in $X[6]$.

2000 Mathematics Subject Classification: 54C45, 54C20, 54B10, 46B99.

Key words and phrases: $C$-embedding, $C^{*}$-embedding, product space, metric space. 
The following theorem will be proved in this paper.

Theorem 1.1. Let $\lambda$ be an infinite cardinal number, $A$ be a $P^{\lambda}$-embedded subset of a topological space $X$, and let $M$ be a metric space. The following conditions are equivalent:

(a) $A \times M$ is $P^{\lambda}$-embedded in $X \times M$.

(b) $A \times M$ is $C^{*}$-embedded in $X \times M$.

(c) $A \times M$ is $U^{\omega}$-embedded in $X \times M$.

Concerning the right place of Theorem 1.1, let us first especially mention the following consequence which provides an affirmative answer to [24, Problem 3] raised by Teodor Przymusiński, and also to [9, Problem 4.14] and [10, Problem 3.1] stated by Takao Hoshina.

Corollary 1.2. Let $X$ be a topological space, $A$ be a subset of $X$, and let $M$ be a non-discrete metric space. Then $A \times M$ is $C$-embedded in $X \times M$ provided it is $C^{*}$-embedded in $X \times M$.

Proof. By Theorem 1.1, it suffices to show that $A$ is $C$-embedded in $X$ if $A \times M$ is $C^{*}$-embedded in $X \times M$. By assumption, $M$ contains an infinite compact subset $K$. By a result of E. Michael (see Lemma 2.1), $A \times K$ is $C^{*}$-embedded in $X \times K$. Finally, by a result of $[20,22]$ (see Lemma 2.3), $A$ is $C$-embedded in $X$.

Theorem 1.1 incorporates also several known results. In case $X \times M$ is an $M$-independent product, Corollary 1.2 coincides with a result of Przymusiński [24]. Let us point out here that the notion of $M$-independence of $X \times M$ is located between $\operatorname{dim}(X \times M)=0$ and $\operatorname{dim} M=0$. In case $M$ is the space of irrational numbers, Corollary 1.2 coincides with a result of $\mathrm{H}$. Ohta [21]. The equivalence between conditions (a) and (b) of Theorem 1.1 was established by K. Yamazaki when $M$ is $\sigma$-locally compact [30], and by T. Hoshina and K. Yamazaki when $M^{2}$ is homeomorphic to $M$ [11].

For a proper understanding of Theorem 1.1, a word should be said also about the last condition (c). The statement that it is equivalent to the previous ones should be compared with Rudin-Starbird's result in [27] that, for a non-discrete metric space $M$, the normality of $X \times M$ is equivalent to the countable paracompactness of $X \times M$. As we shall establish in the next section, the notion of $U^{\omega}$-embedding has a quite nice and useful interpretation in terms of Ishikawa's characterization of countable paracompactness [14].

Another interesting consequence which follows from Theorem 1.1 is the following result.

Corollary 1.3. Let $\lambda$ be an infinite cardinal, $A$ be a $P^{\lambda}$-embedded subset of a topological space $X, Y=K \times M$ be the product of a compact Hausdorff 
space $K$ and a metric space $M$, and let $\mu=\max \{w(K), \lambda\}$. Then $A \times Y$ is $P^{\mu}$-embedded in $X \times Y$ provided it is $C^{*}$-embedded in $X \times Y$.

Proof. Suppose that $(A \times K) \times M$ is $C^{*}$-embedded in $(X \times K) \times M$. Then $A \times K$ is $C^{*}$-embedded in $X \times K$. Hence, it is $P^{\mu}$-embedded in $X \times K$ (see Lemma 2.3). Finally, by Theorem 1.1, $A \times Y$ is $P^{\mu}$-embedded in $X \times Y$.

In view of Corollary 1.3 and Theorem 1.1, the following two problems become natural.

Problem 1. Let $\lambda$ be an infinite cardinal, $A$ be a $P^{\lambda}$-embedded subset of a topological space $X$, and let $Y$ be a closed subspace of the product of a compact Hausdorff space and a metric space, i.e. a paracompact $M$-space in the sense of [18]. Is $A \times Y P^{\lambda}$-embedded in $X \times Y$ if it is $C^{*}$-embedded in $X \times Y$ ?

Problem 2. Let $\lambda$ be an infinite cardinal, and let $A$ be a $P^{\lambda}$-embedded subset of a topological space $X$. Does Theorem 1.1 remain true if $M$ is supposed to be only (i) a Lašnev space, or (ii) a stratifiable space, or (iii) a paracompact $\sigma$-space?

For the notions involved in Problem 2, we refer the reader to [7].

The proof of Theorem 1.1 is divided into a few steps and will be finally accomplished in Section 6. Some of these steps were inspired by ideas stated in the proof of [24, Proposition 5] but, in view of the present situation of arbitrary metric spaces $M$, our arguments are entirely different. The first step of our proof is a general "scattered" reduction of the theorem to the same statement but now only for metric spaces $M$ which are both nowhere locally compact and homogeneous with respect to the weight of their non-empty open subsets. This is done in detail in Section 4 (see Theorem 4.1), where we call the spaces with the second property weight-homogeneous. Next, in Section 5, we deal with the special case of weight-homogeneous and nowhere locally compact spaces $M$, showing that, in this case, controlled simultaneous extension of "many" cozero-sets of $A$ to cozero-sets of $X$ implies (a) of Theorem 1.1 (see Theorem 5.1). Finally, in Section 6, we finish the proof by showing that (b) and (c) of Theorem 1.1 certainly imply the extension property stated in Theorem 5.1 (see Propositions 6.1 and 6.2). The technique developed for the proof of Theorem 1.1 allows one to provide an answer to another question of Przymusiński's [23, Problem 1]. This is done in Theorem 7.1 in the last section.

2. The necessary preliminary results. This section contains all necessary preliminary results which are used in the proof of Theorem 1.1.

For a topological space $X$ and a linear topological space $L$, we use $C(X, L)$ to denote the linear space of all continuous maps from $X$ to $L$. 
Also, as usual, we write $C(X)$ for $C(X, \mathbb{R})$. In what follows, for a map $\varphi$ from a set $T$ to a function space $C(X, L)$, let us agree to write the value of $\varphi$ at a point $t \in T$ as $\varphi[t]$. Suppose that $A \subset X$. A map $\varphi: C(A, L) \rightarrow C(X, L)$ is called a linear extender if it is a linear map and $\varphi[f]$ is an extension of $f$ whenever $f \in C(A, L)$.

The following result is actually due to E. Michael. The proof in the special case of $L=\mathbb{R}$ can be found in Starbird [29].

Lemma 2.1. Let $X$ be a topological space, and let $B$ be a closed subset of a metric space $M$. Then, for every locally convex linear topological space $L$, there exists a linear extender $\varphi: C(X \times B, L) \rightarrow C(X \times M, L)$ such that $\varphi[f](\{x\} \times M)$ is a subset of the the convex hull of $f(\{x\} \times B)$ whenever $x \in X$ and $f \in C(X \times B, L)$.

Outline of proof. With every map $f \in C(X \times B, L)$ and point $x \in X$ we associate another map $f_{x} \in C(B, L)$ defined by $f_{x}(t)=f(x, t), t \in B$. Let $\psi: C(B, L) \rightarrow C(M, L)$ be a Dugundji extender constructed in a standard way [5], i.e. $\psi$ is a linear extender such that, for every $g \in C(B, L)$ and $t \in M$, the point $\psi[g](t)$ belongs to the convex hull of $g(F)$ for some finite $F \subset B$. Then, for every $f \in C(X \times B, L)$, define $\varphi[f] \in C(X \times M, L)$ by setting $\varphi[f](x, t)=\psi\left[f_{x}\right](t),(x, t) \in X \times M$. The map $\varphi: C(X \times B, L) \rightarrow$ $C(X \times M, L)$ so obtained is as required.

A cozero-set in $X$ is a set $U \subset X$ of the form $U=\{x \in X: f(x) \neq 0\}$ for some $f \in C(X)$, and a cozero-set cover of $X$ is a cover of $X$ consisting of cozero-sets in $X$.

Every $\sigma$-locally finite cozero-set cover of a space $X$ has a locally finite cozero-set refinement ([17, Theorem 1.2] and [18, Theorem 1.2]). Modulo this fact, the next lemma was obtained by Morita (see the proof of $[19$, Corollary 10]), while the special case of $P$-embedding is due to Aló and Sennott [1, Corollary 2.4]. For a simple proof, we refer the interested reader to Przymusiński [22, Proposition 3.1].

Lemma 2.2. Let $A$ be a subset of a topological space $X$, and let $\lambda$ be an infinite cardinal. Then $A$ is $P^{\lambda}$-embedded in $X$ if and only if for every locally finite cozero-set cover $\mathcal{V}$ of $A$ of cardinality $|\mathcal{V}| \leq \lambda$, there exists a $\sigma$-locally finite cozero-set cover $\mathcal{W}$ of $X$ such that $\mathcal{V}$ is refined by $\mathcal{W} \cap A=$ $\{W \cap A: W \in \mathcal{W}\}$.

The first statement of the next lemma is due to Aló and Sennott [2]; the second one to Morita and Hoshina [20, Theorem 1.5] and Przymusiński [22, Theorem 3].

Lemma 2.3. Let $\lambda$ be an infinite cardinal, and $A$ be a subset of a topological space $X$. If $A$ is $P^{\lambda}$-embedded in $X$, then $A \times Y$ is $P^{\lambda}$-embedded in $X \times Y$ for every compact Hausdorff space $Y$ with $w(Y) \leq \lambda$. If $Y$ is an 
infinite compact Hausdorff space, then $A$ is $P^{w(Y)}$-embedded in $X$ if and only if $A \times Y$ is $C^{*}$-embedded in $X \times Y$.

A zero-set in $X$ is the complement of a cozero-set in $X$. Concerning the union of zero-sets, we need the following lemma which coincides with $[20$, Lemma 2.3].

Lemma 2.4. Let $\mathcal{Z}$ be a family of zero-sets of a topological space $X$ which admits a locally finite family $\left\{U_{Z}: Z \in \mathcal{Z}\right\}$ of cozero-sets of $X$ with $Z \subset U_{Z}$ for every $Z \in \mathcal{Z}$. Then $\bigcup \mathcal{Z}$ is a zero-set of $X$.

A family $\mathcal{S}$ of subsets of $X$ is uniformly locally finite in $X[12,15]$ if there exists a locally finite cozero-set cover $\mathcal{U}$ of $X$ such that every $U \in \mathcal{U}$ meets at most finitely many members of $\mathcal{S}$.

We complete the list of the preliminary results with the following characterization of $U^{\omega}$-embedding. For a similar characterization, we refer the reader to $[8$, Corollary 1.4$]$.

Lemma 2.5. For a subset $A$ of a topological space $X$, the following conditions are equivalent:

(i) $A$ is $U^{\omega}$-embedded in $X$.

(ii) Every countable family $\mathcal{S}$ of subsets of $A$ which is uniformly locally finite in $A$ is also uniformly locally finite in $X$.

(iii) For every decreasing sequence $\left\{F_{n}: n<\omega\right\}$ of zero-sets of $A$ with $\bigcap\left\{F_{n}: n<\omega\right\}=\emptyset$, there exists a sequence $\left\{Z_{n}: n<\omega\right\}$ of zero-sets of $X$ such that $\bigcap\left\{Z_{n}: n<\omega\right\}=\emptyset$ and $F_{n} \subset Z_{n}, n<\omega$.

Proof. (i) $\Rightarrow$ (ii). Let $\mathcal{S}=\left\{S_{n}: n<\omega\right\}$ be a family of subsets of $A$ which is uniformly locally finite in $A$. By [8, Lemma 1.1], there exists a locally finite family $\left\{G_{n}: n<\omega\right\}$ of cozero-sets of $A$ and a family $\left\{F_{n}: n<\omega\right\}$ of zero-sets of $A$ such that $S_{n} \subset F_{n} \subset G_{n}, n<\omega$. For every $n<\omega$, take a continuous function $f_{n}: A \rightarrow[0, n]$ such that $F_{n} \subset f_{n}^{-1}(n)$ and $A \backslash G_{n} \subset f_{n}^{-1}(0)$. Next, define another continuous function $f: A \rightarrow \mathbb{R}$ by $f=\sum\left\{f_{n}: n<\omega\right\}$. According to (i), there exists $g \in C(X)$ with $f(x) \leq g(x)$ for all $x \in A$. Then $\mathcal{U}=\left\{g^{-1}((-n, n)): n<\omega\right\}$ defines a countable cozero-set cover of $X$ such that each $U \in \mathcal{U}$ meets at most finitely many members of $\mathcal{S}$. Since every countable cozero-set cover has a locally finite cozero-set refinement, $\mathcal{S}$ is uniformly locally finite in $X$.

(ii) $\Rightarrow$ (iii). Let $\left\{F_{n}: n<\omega\right\}$ be a decreasing sequence of zero-sets of $A$ such that $\bigcap\left\{F_{n}: n<\omega\right\}=\emptyset$. Then $\mathcal{V}=\left\{A \backslash F_{n}: n<\omega\right\}$ is a countable cover of $A$ which consists of cozero-sets of $A$ and each member of $\mathcal{V}$ misses some $F_{n}$. Hence, $\left\{F_{n}: n<\omega\right\}$ is uniformly locally finite in $A$ and therefore, by (ii), also in $X$. That is, there exists a locally finite cozero-set cover $\mathcal{U}$ of $X$ such that each $U \in \mathcal{U}$ meets at most finitely many $F_{n}$ 's. Then, for every $n<\omega$, we may set $Z_{n}=\bigcap\left\{X \backslash U: U \in \mathcal{U}\right.$ and $\left.U \cap F_{n}=\emptyset\right\}$. In this way, we 
get a sequence $\left\{Z_{n}: n<\omega\right\}$ of zero-sets of $X$ such that $\bigcap\left\{Z_{n}: n<\omega\right\}=\emptyset$ and $F_{n} \subset Z_{n}, n<\omega$.

(iii) $\Rightarrow$ (i). Let $f \in C(A)$, and let $F_{n}=\left\{x \in A: f(x) \geq 2^{n}\right\}$ for every $n<\omega$. By (iii), there exists a decreasing sequence $\left\{Z_{n}: n<\omega\right\}$ of zero-sets of $X$ such that $\bigcap\left\{Z_{n}: n<\omega\right\}=\emptyset$ and $F_{n} \subset Z_{n}, n<\omega$. Then, for every $n<\omega$, there exists a continuous function $h_{n}: X \rightarrow\left[0,2^{-(n+1)}\right]$ with $Z_{n}=h_{n}^{-1}(0)$. Define a function $h: X \rightarrow \mathbb{R}$ by $h=\sum\left\{h_{n}: n<\omega\right\}$. Since $h \in C(X)$ and $h(x)>0$ for all $x \in X$, we can define another continuous function $g$ on $X$ by setting $g=1 / h$. For $x \in A$, let $n(x)=\min \{n<\omega$ : $\left.x \notin Z_{n}\right\}$. Then $h(x) \leq 2^{-n(x)}$ and, at the same time, $x \notin F_{n(x)}$. That is, $g(x) \geq 2^{n(x)}>f(x)$.

In [8] Hoshina has defined a subset $A$ of $X$ to be $U^{\lambda}$-embedded in $X$ if every uniformly locally finite (in $A$ ) family $\mathcal{F}$ of subsets of $A$ with $|\mathcal{F}| \leq \lambda$ is uniformly locally finite in $X$. The equivalence stated by Lemma 2.5 justifies the use of the same name for the embedding property in the Introduction defined as " $U^{\omega}$-embedding".

3. Subdividing and generating embedding-like properties. We discuss some weaker embedding-like properties which will be used in the next section. A subset $A$ of a topological space $X$ is $z$-embedded in $X$ if each zero-set of $A$ is the restriction to $A$ of a zero-set of $X$. Further, we say that $A$ is well-embedded in $X$ if for every zero-set $Z$ of $X$ with $Z \cap A=\emptyset$, there exists $h \in C(X)$ such that $A \subset h^{-1}(0)$ and $Z \subset h^{-1}(1)$. The following useful characterization of $C$-embedding was proven in [4, Corollary 3.6.B].

Lemma 3.1. A subset $A$ of a topological space $X$ is $C$-embedded in $X$ if and only if it is both z-embedded and well-embedded in $X$.

On the other hand, concerning rectangular subsets of product spaces, we have the following example of well-embedded subsets.

Lemma 3.2. Let $A$ be a subset of a topological space $X, M$ a metric space and let $\mathcal{F}$ be a countable closed cover of $M$ such that $A \times F$ is $C$-embedded in $X \times F$ for every $F \in \mathcal{F}$. Then $A \times M$ is well-embedded in $X \times M$.

Proof. Let $Z$ be a zero-set of $X \times M$ such that $Z \cap(A \times M)=\emptyset$. According to Lemma 3.1, for every $F \in \mathcal{F}$ we can fix an $f_{F} \in C(X \times F)$ such that $A \times F \subset f_{F}^{-1}(0)$ and $Z \cap(X \times F) \subset f_{F}^{-1}(1)$. Then, by Lemma 2.1, we can extend each $f_{F}$ to $g_{F} \in C(X \times M)$ so that $A \times M \subset g_{F}^{-1}(0)$. Finally, $W=\bigcup\left\{g_{F}^{-1}(\mathbb{R} \backslash\{0\}): F \in \mathcal{F}\right\}$ is a cozero-set of $X \times M$ which contains $Z$ and $W \cap(A \times M)=\emptyset$. That is, $A \times M$ is well-embedded in $X \times M$.

As Proposition 3.5 will show, well-embedding plays an important role as a component of another embedding-like property. Following [13], we shall say that $A$ is $C_{1}$-embedded in $X$ if for every zero-set $F$ of $A$ and every zero-set 
$Z$ of $X$ with $F \cap Z=\emptyset$, there exists $h \in C(X)$ such that $F \subset h^{-1}(0)$ and $Z \subset h^{-1}(1)$. It should be mentioned that every $U^{\omega}$-embedded subset is $C_{1}$-embedded and every $C_{1}$-embedded subset is well-embedded while, in general, none of the converse statements hold (see $[8,13]$ ). As a consequence of our results, we shall obtain another example in this direction which is related to rectangular subsets of product spaces (see Example 3.4). Our special interest in $C_{1}$-embedding is inspired by the following property.

Lemma 3.3. Let $\lambda$ be an infinite cardinal, $A$ be a $C_{1}$-embedded subset of a topological space $X$, and let $\mathcal{F}$ be a countable family of zero-sets of $X$ which covers $A$ and $F \cap A$ is $P^{\lambda}$-embedded in $X$ for each $F \in \mathcal{F}$. Then $A$ is also $P^{\lambda}$-embedded in $X$.

Proof. Let $\mathcal{U}$ be a locally finite cozero-set cover of $A$ with $|\mathcal{U}| \leq \lambda$. For every $F \in \mathcal{F}$, by hypothesis and Lemma 2.2, there exists a $\sigma$-locally finite cozero-set cover $\left\{G_{(U, F)}: U \in \mathcal{U}\right\}$ of $X$ with $G_{(U, F)} \cap(F \cap A) \subset U$ whenever $U \in \mathcal{U}$. Hence, for every $F \in \mathcal{F}$, there also exists a zero-set cover $\left\{Z_{(U, F)}: U \in \mathcal{U}\right\}$ of $X$ and a cozero-set one $\left\{V_{(U, F)}: U \in \mathcal{U}\right\}$ such that $V_{(U, F)} \subset Z_{(U, F)} \subset G_{(U, F)}, U \in \mathcal{U}$. Since $A$ is $C_{1}$-embedded in $X$, for every $(U, F) \in \mathcal{U} \times \mathcal{F}$ there now exists a cozero-set $W_{(U, F)}$ of $X$ such that $W_{(U, F)} \cap\left(\left(Z_{(U, F)} \cap A\right) \backslash U\right)=\emptyset$ and $F \subset W_{(U, F)}$. Then $\mathcal{W}_{0}=\left\{V_{(U, F)} \cap W_{(U, F)}\right.$ : $(U, F) \in \mathcal{U} \times \mathcal{F}\}$ is a $\sigma$-locally finite and cozero-set (in $X$ ) cover of $A$ such that $\mathcal{U}$ is refined by $\mathcal{W}_{0} \cap A$. Since $A$ is $C_{1}$-embedded in $X$ and $W_{0}=\bigcup \mathcal{W}_{0}$ is a cozero-set of $X$ which contains $A$, there exists a cozero-set $W_{1}$ of $X$ such that $W_{1} \cap A=\emptyset$ and $X \backslash W_{0} \subset W_{1}$. Hence, $\mathcal{W}=\mathcal{W}_{0} \cup\left\{W_{1}\right\}$ is a $\sigma$-locally finite cozero-set cover of $X$ such that $\mathcal{U}$ is refined by $\mathcal{W} \cap A$. Therefore, by Lemma $2.2, A$ is $P^{\lambda}$-embedded in $X$.

We are now ready for the following example which shows that " $U$ " embedded" cannot be weakened to "well-embedded" in condition (c) of Theorem 1.1.

EXAmple 3.4. There exist a normal space $X$, a closed subset $A$ of $X$, and a metric space $M$ such that $A \times M$ is well-embedded in $X \times M$ but not $C_{1}$-embedded.

Pr o o f. According to $[25,26]$, there exists a normal space $X$ and a closed subset $A$ of $X$ such that $A \times J(\omega)$ is not $C$-embedded in $X \times J(\omega)$. Here, $J(\omega)$ is the metrizable hedgehog with $\omega$ spines. Note that $J(\omega)$ admits a countable cover $\mathcal{F}$ of compact subsets. Since $A$ is $C$-embedded in $X$, it follows from Lemma 2.3 that each $A \times F, F \in \mathcal{F}$, is $C$-embedded in $X \times F$ and hence, by Lemma 2.1, in $X \times J(\omega)$ as well. Therefore, by Lemma 3.2, $A \times J(\omega)$ is well-embedded in $X \times J(\omega)$ while, by Lemma 3.3 , it is not $C_{1}$-embedded in $X \times J(\omega)$. 
In what follows, we say that a subset $A$ is $C U$-embedded in $X$ if for every zero-set $F$ of $A$ and every zero-set $Z$ of $X$ with $Z \cap F=\emptyset$, there exists $h \in C(X)$ such that $F \subset h^{-1}(0)$ and $Z \cap A \subset h^{-1}(1)$. According to the definitions of well-embedding and $C_{1}$-embedding, we now get the following immediate proposition.

Proposition 3.5. A subset $A$ of a topological space $X$ is $C_{1}$-embedded in $X$ if and only if it is both $C U$-embedded and well-embedded in $X$.

We conclude this section with the following important example of $C U$ embedded subsets.

Proposition 3.6. Let $A$ be a subset of a topological space $X$ which is either $C^{*}$-embedded or $U^{\omega}$-embedded in $X$. Then $A$ is $C U$-embedded in $X$.

Proof. In case $A$ is $C^{*}$-embedded in $X$, this is obvious. On the other hand, every $U^{\omega}$-embedded subset is $C_{1}$-embedded as we have stated before Lemma 3.3. Hence, in case $A$ is $U^{\omega}$-embedded, this follows from Proposition 3.5 .

4. A reduction to "nice" metric factors. Here, we show how the proof of Theorem 1.1 can be reduced to metric spaces $M$ which are weighthomogeneous, i.e. $w(U)=w(M)$ for every non-empty open subset $U$ of $M$. The following theorem, which may have some independent interest, will be proved.

Theorem 4.1. Let $X$ be a topological space, $M$ be a metric space, $\lambda$ be an infinite cardinal, and let $A$ be a $P^{\lambda}$-embedded subset of $X$ such that $A \times M$ is $C U$-embedded in $X \times M$. Also, let $A \times S$ be $P^{\lambda}$-embedded in $X \times S$ for every weight-homogeneous and nowhere locally compact closed subspace $S$ of $M$. Then $A \times M$ is $P^{\lambda}$-embedded in $X \times M$.

To prepare for the proof of Theorem 4.1, we need a bit more terminology. For a space $Y$ we use $\mathcal{P}(Y)$ to denote the set of all subsets of $Y$, and $\mathcal{C}(Y)$ to denote that of all closed subsets of $Y$. Also, we write $\mathcal{T}(Y)$ for the topology of $Y$. Finally for $S \subset Y$ and $U \subset S$, we denote by $\operatorname{cl}_{S}(U)$ the closure of $U$ in $S$.

Suppose that $S \in \mathcal{P}(Y)$ and $\mathcal{P} \subset \mathcal{P}(Y)$. By transfinite induction, for each ordinal $\alpha$ we define a subset $S^{(\mathcal{P}, \alpha)}$ of $S$ by setting $S^{(\mathcal{P}, 0)}=S$ and, for $\alpha>0$,

$$
S^{(\mathcal{P}, \alpha)}=S \backslash \bigcup\left\{U \in \mathcal{T}(S): \operatorname{cl}_{S}(U) \cap S^{(\mathcal{P}, \beta)} \in \mathcal{P} \text { for some } \beta<\alpha\right\} .
$$

We say that $S$ is $\mathcal{P}$-scattered if $S^{(\mathcal{P}, \alpha)}=\emptyset$ for some $\alpha$. For such $S$ we also define the $\mathcal{P}$-scattered height $\kappa_{\mathcal{P}}(S)$ as the first ordinal $\alpha$ with $S^{(\mathcal{P}, \alpha)}=\emptyset$. Sometimes, for convenience, we write $S^{(\alpha)}$ instead of $S^{(\mathcal{P}, \alpha)}$. 
Lemma 4.2. Let $S$ be a subset of a metric space $M$, and let $\mathcal{P} \subset \mathcal{P}(M)$ be such that $\mathcal{P}(P) \subset \mathcal{P}$ whenever $P \in \mathcal{P}$. Then for each ordinal $\alpha$ the following holds:

(1) $S^{(\alpha)}$ is closed in $S$.

(2) If $U \in \mathcal{T}(S)$, then $U^{(\alpha)} \subset S^{(\alpha)}$.

(3) $S \backslash S^{(\alpha)}$ is $\mathcal{P}$-scattered.

Proof. (1) follows from the definition of $S^{(\alpha)}$ while (3) is a consequence of (1) and (2). So, it remains to prove (2). Suppose, on the contrary, that (2) fails for some $U \in \mathcal{T}(S)$, and let $\alpha$ be the first ordinal such that $U^{(\alpha)} \backslash S^{(\alpha)}$ $\neq \emptyset$. Since $U^{(0)}=U \subset S=S^{(0)}$, it follows that $\alpha>0$. Then, by definition, there exists $G \in \mathcal{T}(S)$ such that $G \cap U^{(\alpha)} \neq \emptyset$ and $\operatorname{cl}_{S}(G) \cap S^{(\beta)} \in \mathcal{P}$ for some $\beta<\alpha$. Take $H \in \mathcal{T}(G)$ such that $H \cap U^{(\alpha)} \neq \emptyset$ and $\operatorname{cl}_{S}(H) \subset U$. Then $\operatorname{cl}_{S}(H) \cap U^{(\beta)} \in \mathcal{C}\left(\operatorname{cl}_{S}(H)\right)$ because $\operatorname{cl}_{S}(H) \subset U$ and $U^{(\beta)}$ is closed in $U$. On the other hand, $U^{(\beta)} \subset S^{(\beta)}$ because $\beta<\alpha$. Therefore,

$$
\operatorname{cl}_{S}(H) \cap U^{(\beta)} \in \mathcal{C}\left(\operatorname{cl}_{S}(H) \cap S^{(\beta)}\right) \subset \mathcal{C}\left(\operatorname{cl}_{S}(G) \cap S^{(\beta)}\right) \subset \mathcal{P} .
$$

However, this is impossible because $H \cap U^{(\alpha)} \neq \emptyset$.

Suppose that $S \subset M$ and $\mathcal{P} \subset \mathcal{P}(M)$. We say that a family $\mathcal{F}$ of subsets of $S$ is a $\sigma$-discrete closed $\mathcal{P}$-cover of $S$ if $\mathcal{F}$ is a $\sigma$-discrete (in $S$ ) cover of $S$ such that $\mathcal{F} \subset \mathcal{P} \cap \mathcal{E}(S)$.

Lemma 4.3. Let $M$ be a metric space, and let $\mathcal{P} \subset \mathcal{P}(M)$ be such that $\emptyset \in \mathcal{P}$ and $\mathcal{C}(P) \subset \mathcal{P}$ whenever $P \in \mathcal{P}$. Then every $\mathcal{P}$-scattered subspace $S$ of $M$ has a $\sigma$-discrete closed $\mathcal{P}$-cover.

Proof (by transfinite induction on $\kappa_{\mathcal{P}}(S)$ of the $\mathcal{P}$-scattered subspaces $S$ of $M$ ). If $\kappa_{\mathcal{P}}(S)=0$, then $S=\emptyset$ and $\{S\}$ is a $\sigma$-discrete closed $\mathcal{P}_{-}$ cover of $S$. Suppose that, for some $\alpha>0$, every $\mathcal{P}$-scattered subspace $S$ of $M$ with $\kappa_{\mathcal{P}}(S)<\alpha$ has a $\sigma$-discrete closed $\mathcal{P}$-cover. Next, let $T \subset M$ be such that $\kappa_{\mathcal{P}}(T)=\alpha$. For every $\beta<\alpha$ we set $U_{\beta}=T \backslash T^{(\beta)}$. Note that $\kappa_{\mathcal{P}}\left(U_{\beta}\right) \leq \beta$ because, by Lemma $4.2, U_{\beta}^{(\beta)} \subset T^{(\beta)} \cap U_{\beta}=\emptyset$. Hence, by the inductive hypothesis, for every $\beta<\alpha$ there exists a $\sigma$-discrete closed $\mathcal{P}$-cover $\mathcal{G}_{\beta}$ of $U_{\beta}$. On the other hand, the open family $\left\{U_{\beta}: \beta<\alpha\right\}$ has a $\sigma$-discrete closed (in $T$ ) refinement $\mathcal{L}$ with $\bigcup \mathcal{L}=\bigcup\left\{U_{\beta}: \beta<\alpha\right\}$ because $M$ is a metric space. For every $L \in \mathcal{L}$, pick a fixed $\beta(L)<\alpha$ such that $L \subset U_{\beta(L)}$. Then $\mathcal{D}=\left\{L \cap G: L \in \mathcal{L}\right.$ and $\left.G \in \mathcal{G}_{\beta(L)}\right\}$ is a $\sigma$-discrete closed (in $T$ ) family such that $\mathcal{D} \subset \mathcal{P}$ because each $D \in \mathcal{D}$ is a subset of some $G \in \bigcup\left\{\mathcal{G}_{\beta}: \beta<\alpha\right\} \subset \mathcal{P}$. Now, let $T_{\alpha}=T \backslash \bigcup \mathcal{D}=\bigcap\left\{T^{(\beta)}: \beta<\alpha\right\}$. Since $T^{(\alpha)}=\emptyset$, by definition, there exists a cover $\mathcal{H} \subset \mathcal{T}(T)$ of $T_{\alpha}$ such that to every $H \in \mathcal{H}$ there corresponds a $\beta(H)<\alpha$ with $\operatorname{cl}_{T}(H) \cap T^{(\beta(H))} \in \mathcal{P}$. Hence, in particular, $\operatorname{cl}_{T}(H) \cap T_{\alpha} \in \mathcal{P}$ because $T_{\alpha}$ is closed in $T^{(\beta(H))}$. Then, let $\mathcal{E} \subset \mathcal{C}\left(T_{\alpha}\right)$ be a $\sigma$-discrete (in $T$ ) cover of $T_{\alpha}$ which refines $\mathcal{H}$. This 
certainly implies that $\mathcal{E} \subset \mathcal{P}$. Therefore, $\mathcal{F}=\mathcal{D} \cup \mathcal{E}$ is a $\sigma$-discrete closed $\mathcal{P}$-cover of $T$.

Proof of Theorem 4.1. Let $X, A, \lambda$ and $M$ be as in that theorem. Also, let

$$
\mathcal{P}=\left\{S \in \mathcal{P}(M): A \times S \text { is } P^{\lambda} \text {-embedded in } X \times S\right\} .
$$

The family $\mathcal{P}$ has the following two important properties.

(i) $\emptyset \in \mathcal{P}$ and $\mathcal{C}(S) \subset \mathcal{P}$ whenever $S \in \mathcal{P}$.

(ii) $\bigcup \mathcal{D} \in \mathcal{P}$ whenever $\mathcal{D} \subset \mathcal{P}$ is discrete in $\bigcup \mathcal{D}$.

Indeed, the empty set is $P$-embedded in any space and, in particular, $\emptyset \in \mathcal{P}$. The rest of (i) follows from Lemma 2.1. As for (ii), it follows from the fact that, in this case, the family $\{X \times D: D \in \mathcal{D}\}$ is discrete in $X \times \bigcup \mathcal{D}$.

The conclusion of our theorem becomes now equivalent to the statement that $M \in \mathcal{P}$. Suppose on the contrary that $M \notin \mathcal{P}$, and let $S \in \mathcal{C}(M) \backslash \mathcal{P}$ be such that $w(S)=\min \{w(F): F \in \mathcal{C}(M) \backslash \mathcal{P}\}$. Let us show that

(iii) $S$ has a countable closed cover $\mathcal{F}$ with $\mathcal{F} \subset \mathcal{P}$.

According to (i), (ii) and Lemma 4.3, it suffices to show that $S$ is a $\mathcal{P}_{-}$ scattered subspace of $M$. Suppose on the contrary that we can find $\gamma$ such that $S^{(\mathcal{P}, \gamma)}=S^{(\mathcal{P}, \gamma+1)} \neq \emptyset$. Then, by Lemma $2.3, S^{(\mathcal{P}, \gamma)}$ is nowhere locally compact while, by the choice of $S, S^{(\mathcal{P}, \gamma)}$ is weight-homogeneous. Moreover, $S^{(\mathcal{P}, \gamma)}$ is closed in $M$ by Lemma 4.2 . Hence, by hypothesis of our theorem, $S^{(\mathcal{P}, \gamma)} \in \mathcal{P}$ and therefore, by definition, $S^{(\mathcal{P}, \gamma+1)}=\emptyset$. The contradiction so obtained completes the verification of (iii).

We now complete the proof as follows. By Lemma 3.2, (iii) implies that $A \times S$ is well-embedded in $X \times S$. On the other hand, $A \times S$ is a zero-set of $A \times M$ and $X \times S$ is a zero-set of $X \times M$. Thus, it follows from Lemma 2.1 that every zero-set of $A \times S$ is a zero-set of $A \times M$ and every zero-set of $X \times S$ is a zero-set of $X \times M$. Hence, $A \times S$ is also $C U$-embedded in $X \times S$ because, by hypothesis, $A \times M$ is $C U$-embedded in $X \times M$. Then, by Proposition 3.5, $A \times S$ is $C_{1}$-embedded in $X \times S$. Moreover, it follows from Lemma 2.1 again that $A \times F$ is $P^{\lambda}$-embedded in $X \times S$ for each $F \in \mathcal{F}$. However, according to (iii) and Lemma 3.3, these finally imply that $S \in \mathcal{P}$, which contradicts the choice of $S$.

5. A further reduction of separating the factors. For sets $D$ and $R$, we use $R^{D}$ to denote the set of all maps from $D$ to $R$ and, in the special case of $R=2$, we identify $2^{D}$ with the power $\mathcal{P}(D)$ of $D$. If $T$ is a set, $B \subset R$ and $\mathcal{G}: T \rightarrow \mathcal{P}(B)^{D}$, then we say that $\mathcal{H}: T \rightarrow \mathcal{P}(R)^{D}$ is an expansion of $\mathcal{G}$ provided $\mathcal{G}[t](d) \subset \mathcal{H}[t](d)$ for every $t \in T$ and $d \in D$. Finally, with every map $\mathcal{H}: T \rightarrow \mathcal{P}(R)^{D}$ we associate another map $\langle\mathcal{H}, D\rangle: T \rightarrow \mathcal{P}(R)$ defined by $\langle\mathcal{H}, D\rangle(t)=\bigcup \mathcal{H}[t](D)$ for every $t \in T$. 
As usual, an ordinal is the set of smaller ordinals and a cardinal is an initial ordinal. For cardinals $\varkappa$ and $\mu$, let $\varkappa^{<\mu}=\bigcup\left\{\varkappa^{\delta}: \delta<\mu\right\}$. For convenience, we regard $\varkappa^{0}$ as the singleton $\{\emptyset\}$. With every $\sigma \in \varkappa^{\delta}$ and $\alpha<\varkappa$ we associate another map $\sigma^{\wedge} \alpha \in \varkappa^{\delta+1}$ defined by $\sigma^{\wedge} \alpha \mid \delta=\sigma$ and $\sigma^{\wedge} \alpha(\delta)=\alpha$. We say that a map $\mathcal{H}: \varkappa^{<\omega} \rightarrow \mathcal{P}(R)^{D}$ is decreasing if, for every $\sigma \in \varkappa^{<\omega}$ and $\alpha<\varkappa$, the family $\mathcal{H}\left[\sigma^{\wedge} \alpha\right](D)$ refines $\mathcal{H}[\sigma](D)$, i.e. each member of $\mathcal{H}\left[\sigma^{\wedge} \alpha\right](D)$ is included in some member of $\mathcal{H}[\sigma](D)$.

We also need the following terminology. If $Y$ and $M$ are topological spaces and $\mathcal{S}: \varkappa^{<\omega} \rightarrow \mathcal{P}(M)$, then we say that $\mathcal{H}: \varkappa^{<\omega} \rightarrow \mathcal{P}(Y)^{\varkappa}$ is $\mathcal{S}$-free provided

$$
\bigcap\left\{\operatorname{cl}_{Y}(\langle\mathcal{H}, \varkappa\rangle(t \mid n)) \times \operatorname{cl}_{M}(\mathcal{S}(t \mid n)): n<\omega\right\}=\emptyset \quad \text { for every } t \in \varkappa^{\omega} .
$$

We say that a map $\mathcal{S}: \varkappa^{<\omega} \rightarrow \mathcal{T}(M)$ is a sieve on $M$ if $\mathcal{S}(\emptyset)=M$ and $\mathcal{S}(\sigma)=\bigcup\left\{\mathcal{S}\left(\sigma^{\wedge} \alpha\right): \alpha<\varkappa\right\}$ for every $\sigma \in \varkappa^{<\omega}$. For a sieve $\mathcal{S}: \varkappa^{<\omega} \rightarrow \mathcal{T}(M)$ on a space $M$ and a point $z \in M$, let

$$
\begin{aligned}
& \Sigma_{n}(z)=\left\{\sigma \in \varkappa^{n}: z \in \operatorname{cl}_{M}(\mathcal{S}(\sigma))\right\}, \quad n<\omega, \\
& \Sigma_{\omega}(z)=\left\{t \in \varkappa^{\omega}: t \mid n \in \Sigma_{n}(z) \text { for every } n<\omega\right\},
\end{aligned}
$$

and

$$
\mathcal{S}_{n}(z)=\bigcup\left\{\mathcal{S}(\sigma): \sigma \in \Sigma_{n}(z)\right\}, \quad n<\omega .
$$

We say that a sieve $\mathcal{S}: \varkappa^{<\omega} \rightarrow \mathcal{T}(M)$ is strong if

(i) $\emptyset \notin \mathcal{S}\left(\varkappa^{<\omega}\right)$,

(ii) each family $\left\{\mathcal{S}(\sigma): \sigma \in \varkappa^{n}\right\}, n<\omega$, is locally finite in $M$, and in $M$.

(iii) whenever $z \in M$, the collection $\left\{\mathcal{S}_{n}(z): n<\omega\right\}$ is a local base at $z$

Finally, for a space $Y$, we write $\mathcal{T}_{0}(Y)$ for the collection of all cozero-sets of $Y$, and $\mathrm{e}_{0}(Y)$ for that of the zero-sets of $Y$.

TheOREm 5.1. Let $\lambda$ be an infinite cardinal, $A$ be a $P^{\lambda}$-embedded subset of a topological space $X$, and let $M$ be a weight-homogeneous nowhere locally compact metric space. Also, let $\varkappa=w(M)$. Then $A \times M$ is $P^{\lambda}$-embedded in $X \times M$ provided for every strong sieve $\mathcal{S}: \varkappa^{<\omega} \rightarrow \mathcal{T}(M)$ on $M$, every decreasing $\mathcal{S}$-free map $\mathcal{G}: \varkappa^{<\omega} \rightarrow \mathcal{T}_{0}(A)^{\varkappa}$ admits an $\mathcal{S}$-free expansion $\mathcal{H}:$ $\varkappa^{<\omega} \rightarrow \mathcal{T}_{0}(X)^{\varkappa}$.

To prepare for the proof of Theorem 5.1, we arrange a few preliminary facts into separate statements.

Lemma 5.2. Let $\varkappa$ be a cardinal number, $M$ and $Y$ be topological spaces, $\mathcal{S}: \varkappa^{<\omega} \rightarrow \mathcal{T}(M)$ be a strong sieve on $M$, and let $\mathcal{G}: \varkappa^{<\omega} \rightarrow \mathcal{P}(Y)^{\varkappa}$ be a decreasing map. For every $z \in M$ the following two conditions are equivalent: 
(a) $\bigcap\left\{\operatorname{cl}_{Y}(\langle\mathcal{G}, \varkappa\rangle(t \mid n)): n<\omega\right\}=\emptyset$ for every $t \in \Sigma_{\omega}(z)$.

(b) $\bigcap\left\{\bigcup\left\{\operatorname{cl}_{Y}(\langle\mathcal{G}, \varkappa\rangle(\sigma)): \sigma \in \Sigma_{n}(z)\right\}: n<\omega\right\}=\emptyset$.

Proof. Let $y \in Y$ be such that for every $n<\omega$ there is $\sigma \in \Sigma_{n}(z)$ with $y \in \operatorname{cl}_{Y}(\langle\mathcal{G}, \varkappa\rangle(\sigma))$. Then, for every $n<\omega$, we may define a non-empty subset $\Sigma_{n}$ of $\Sigma_{n}(z)$ by $\Sigma_{n}=\left\{\sigma \in \Sigma_{n}(z): y \in \operatorname{cl}_{Y}(\langle\mathcal{G}, \varkappa\rangle(\sigma))\right\}$. Note that $\sigma \in \Sigma_{n+1}$ implies $\sigma \mid n \in \Sigma_{n}$ because $\mathcal{G}$ is decreasing. In addition, each $\Sigma_{n}$ is finite because so is $\Sigma_{n}(z)$. Hence, by König's Lemma (see Lemma 5.7 in Chapter II of [16]), there exists a point $t \in \varkappa^{\omega}$ such that $t \mid n \in \Sigma_{n} \subset \Sigma_{n}(z)$ for every $n<\omega$. In particular, $t \in \Sigma_{\omega}(z)$ and $y \in \bigcap\left\{\operatorname{cl}_{Y}(\langle\mathcal{G}, \varkappa\rangle(t \mid n)): n<\omega\right\}$, which demonstrates that (a) implies (b). Since the converse implication is obvious, this completes the proof.

We now turn to statements which concern relationships between the basic concepts involved in our Theorem 5.1. The first one sheds a bit more light on $\mathcal{S}$-free maps and their role as components of embedding-like properties in product spaces. It will be found to be very useful in the next section.

Lemma 5.3. Let $\varkappa$ be a cardinal number, $M$ and $Y$ be topological spaces, $\mathcal{S}: \varkappa^{<\omega} \rightarrow \mathcal{T}(M)$ be a strong sieve on $M$, and let $\mathcal{G}: \varkappa^{<\omega} \rightarrow \mathcal{P}(Y)^{\varkappa}$ be a decreasing map. Then $\mathcal{G}$ is $\mathcal{S}$-free if and only if the family $\{\langle\mathcal{G}, \varkappa\rangle(\sigma) \times \mathcal{S}(\sigma)$ : $\left.\sigma \in \varkappa^{<\omega}\right\}$ is locally finite in $Y \times M$.

Proof. Suppose that $\mathcal{G}$ is $\mathcal{S}$-free, and let $(y, z) \in Y \times M$. Then, by definition, $\bigcap\left\{\operatorname{cl}_{Y}(\langle\mathcal{G}, \varkappa\rangle(t \mid n)): n<\omega\right\}=\emptyset$ for every $t \in \Sigma_{\omega}(z)$. According to Lemma 5.2, this implies that

$$
\bigcap\left\{\bigcup\left\{\operatorname{cl}_{Y}(\langle\mathcal{G}, \varkappa\rangle(\sigma)): \sigma \in \Sigma_{n}(z)\right\}: n<\omega\right\}=\emptyset .
$$

Hence, there exists $m<\omega$ and a neighbourhood $V$ of $y$ in $Y$ such that

$$
V \cap\langle\mathcal{G}, \varkappa\rangle(\sigma)=\emptyset \text { for every } \sigma \in \Sigma_{m}(z) .
$$

We now consider the set

$$
\mathcal{L}_{m}(z)=M \backslash \bigcup\left\{\operatorname{cl}_{M}(\mathcal{S}(\sigma)): \sigma \in \varkappa^{m} \backslash \Sigma_{m}(z)\right\} .
$$

Thus, we get a neighbourhood $\mathcal{L}_{m}(z)$ of $z$ such that

$$
\Sigma_{m}(x) \subset \Sigma_{m}(z) \quad \text { for every } x \in \mathcal{L}_{m}(z) .
$$

Whenever $n \geq m$ and $\tau \in \varkappa^{n}$, note that, by (5.3), $x \in \mathcal{S}(\tau) \cap \mathcal{L}_{m}(z)$ implies $\tau \mid m \in \Sigma_{m}(x) \subset \Sigma_{m}(z)$. Therefore, $\mathcal{S}(\tau) \cap \mathcal{L}_{m}(z) \neq \emptyset$ implies $\tau \mid m \in$ $\Sigma_{m}(z)$. Since $\mathcal{G}$ is decreasing, by (5.1), this finally implies that $\left(V \times \mathcal{L}_{m}(z)\right) \cap$ $(\langle\mathcal{G}, \varkappa\rangle(\tau) \times \mathcal{S}(\tau))=\emptyset$ for every $\tau \in \varkappa^{n}$ with $n \geq m$. Since each family $\left\{\mathcal{S}(\sigma): \sigma \in \varkappa^{n}\right\}$ is locally finite, $z$ has a neighbourhood $U$ in $M$ such that $U \subset \mathcal{L}_{m}(z)$ and $\left\{\sigma \in \varkappa^{<m}: U \cap \mathcal{S}(\sigma) \neq \emptyset\right\}$ is finite. Then $V \times U$ is a neighbourhood of $(y, z)$ in $Y \times M$ which meets only finitely many members of $\left\{\langle\mathcal{G}, \varkappa\rangle(\sigma) \times \mathcal{S}(\sigma): \sigma \in \varkappa^{<\omega}\right\}$. 
To show the converse, take a point $z \in M$ and $t \in \Sigma_{\omega}(z)$. Since the family $\left\{\langle\mathcal{G}, \varkappa\rangle(\sigma) \times \mathcal{S}(\sigma): \sigma \in \varkappa^{<\omega}\right\}$ is locally finite, for every $y \in Y$ there exists a neighbourhood of $(y, z)$ in $Y \times M$ which meets only finitely many members of $\{\langle\mathcal{G}, \varkappa\rangle(t \mid n) \times \mathcal{S}(t \mid n): n<\omega\}$. This certainly implies that $y \notin \operatorname{cl}_{Y}(\langle\mathcal{G}, \varkappa\rangle(t \mid m))$ for some $m<\omega$, i.e. $\bigcap\left\{\operatorname{cl}_{Y}(\langle\mathcal{G}, \varkappa\rangle(t \mid n)): n<\omega\right\}=\emptyset$, which completes the proof.

Our next lemmas are related to the existence of strong sieves on weighthomogeneous nowhere locally compact metric spaces.

LEMMA 5.4. Let $M$ be a weight-homogeneous non-compact metric space. Then there exists a family $\mathcal{U}$ of non-empty open subsets of $M$ which is discrete in $M$ and $|\mathcal{U}|=w(M)$.

Proof. Since $M$ has a $\sigma$-discrete base, the proof reduces to the case of $w(M)=\varkappa>\omega$ and $\operatorname{cf}(\varkappa)=\omega$. In this case, there exists a sequence $\left\{\varkappa_{n}: n<\omega\right\}$ of regular cardinals such that $\varkappa=\sup \varkappa_{n}$. Since $M$ is not compact, there is a discrete (in $M$ ) family $\left\{U_{n}: n<\omega\right\}$ of non-empty open subsets of $M$. Since $M$ is weight-homogeneous, $w\left(U_{n}\right)=\varkappa>\varkappa_{n}$ for each $n<\omega$. Hence, for every $n<\omega$, there is a family $\mathcal{U}_{n}$ of non-empty open subsets of $U_{n}$ which is discrete in $\operatorname{cl}_{M}\left(U_{n}\right)$ and $\left|\mathcal{U}_{n}\right| \geq \varkappa_{n}$. Finally, the family $\mathcal{U}=\bigcup\left\{\mathcal{U}_{n}: n<\omega\right\}$ is discrete in $M$ and $|\mathcal{U}|=\varkappa$.

Lemma 5.4 fails if $M$ is not weight-homogeneous. For example, endow $M=\omega_{\omega}+1$ with the following topology. A basic neighbourhood of the point $\omega_{\omega}$ is a set of the form $M \backslash \omega_{n}$ for $n<\omega$, while each point of $M \backslash\left\{\omega_{\omega}\right\}$ is isolated. Then $M$ is a metric space with $w(M)=\omega_{\omega}$ but the cardinality of any discrete (in $M$ ) family $\mathcal{U}$ of open subsets of $M$ is less than $\omega_{\omega}$. This was kindly pointed out by S. Fujii.

Lemma 5.5. Let $M$ be a weight-homogeneous nowhere locally compact metric space, and let $\varkappa=w(M)$. Then there exists a strong sieve $\mathcal{S}: \varkappa^{<\omega} \rightarrow$ $\mathcal{T}(M)$ on $M$.

Pr o of (by induction on $n<\omega$ ). Take a compatible metric $d$ on $M$ such that $d(y, z) \leq 1$ for every $y, z \in M$. Next, set $\mathcal{S}(\emptyset)=M$, and then suppose that $\mathcal{S}(\sigma)$ is a non-empty open subset of $M$ for some $\sigma \in \varkappa^{n}$ and $n<\omega$. Let $D$ be a non-empty open subset of $\mathcal{S}(\sigma)$ with $\operatorname{diam}_{d}(D)<1 / 2^{n+1}$. According to Lemma 5.4 , there exists a discrete (in $M$ ) family $\mathcal{U}$ of non-empty open subsets of $D$ such that $|\mathcal{U}|=\varkappa$. On the other hand, there exists a locally finite (in $M$ ) family $\mathcal{V}$ of non-empty open subsets of $M$ such that $\bigcup \mathcal{V}=\mathcal{S}(\sigma)$ and $\operatorname{diam}_{d}(V)<1 / 2^{n+1}, V \in \mathcal{V}$. Since $|\mathcal{U} \cup \mathcal{V}|=\varkappa$, we may now fix a bijection $\mathcal{S}_{\sigma}: \varkappa \rightarrow \mathcal{U} \cup \mathcal{V}$. Then, for every $\alpha<\varkappa$, just set $\mathcal{S}\left(\sigma^{\wedge} \alpha\right)=\mathcal{S}_{\sigma}(\alpha)$. In this way, we get a sieve $\mathcal{S}: \varkappa^{<\omega} \rightarrow \mathcal{T}(M)$ such that $\emptyset \notin \mathcal{S}\left(\varkappa^{<\omega}\right)$ and $\operatorname{diam}_{d}\left(\operatorname{cl}_{M}(\mathcal{S}(\sigma))\right) \leq 1 / 2^{n}$ whenever $\sigma \in \varkappa^{n}$. Also, $\left\{\mathcal{S}(\sigma): \sigma \in \varkappa^{n}\right\}$ is locally finite for every $n<\omega$. That is, $\mathcal{S}$ is a strong sieve on $M$. 
It should be mentioned that a metric space $M$ which has a strong sieve $\mathcal{S}: \varkappa^{<\omega} \rightarrow \mathcal{T}(M)$ for some $\varkappa \geq \omega$ is weight-homogeneous and nowhere locally compact. This fact will not be used in the paper and we leave the arguments to the interested reader.

We conclude the preparation for the proof of Theorem 5.1 with another lemma concerning special partitions of unity subordinate to strong sieves. Following the notation of Section 2, let $C(M, \mathbb{I})$ denote the subset of $C(M)$ of all continuous functions on $M$ with values in $\mathbb{I}=[0,1]$. We say that a map $\xi: \varkappa^{<\omega} \rightarrow C(M, \mathbb{I})$ is a sieve-partition of unity on $M$ if $\xi[\emptyset]$ is the constant function on $M$ with value 1 , and $\xi[\sigma]=\sum\left\{\xi\left[\sigma^{\wedge} \alpha\right]: \alpha<\varkappa\right\}$ for every $\sigma \in \varkappa^{<\omega}$. For $h \in C(M, \mathbb{I})$, denote by $\operatorname{supp}(h)$ the supporting set of $h$, i.e. $\operatorname{supp}(h)=\operatorname{cl}_{M}\left(h^{-1}((0,1])\right)$.

Lemma 5.6. Let $\varkappa$ be an infinite cardinal, and let $\mathcal{S}: \varkappa^{<\omega} \rightarrow \mathcal{T}_{0}(M)$ be a strong sieve on a metric space $M$. Then there exists a sieve-partition of unity $\xi: \varkappa^{<\omega} \rightarrow C(M, \mathbb{I})$ on $M$ such that $\operatorname{supp}(\xi[\sigma]) \subset \mathcal{S}(\sigma)$ for every $\sigma \in \varkappa^{<\omega}$.

Pr o o f. Since each $\left\{\mathcal{S}\left(\sigma^{\wedge} \alpha\right): \alpha<\varkappa\right\}, \sigma \in \varkappa^{<\omega}$, is a locally finite cozeroset cover of $\mathcal{S}(\sigma)$, there exists a sieve $\mathcal{L}: \varkappa^{<\omega} \rightarrow \mathcal{T}_{0}(M)$ on $M$ such that $\operatorname{cl}_{M}(\mathcal{L}(\sigma)) \subset \mathcal{S}(\sigma)$ for every $\sigma \in \varkappa^{<\omega}$. Take a map $\eta: \varkappa^{<\omega} \rightarrow C(M, \mathbb{I})$ such that $\eta[\sigma]^{-1}(0)=M \backslash \mathcal{L}(\sigma)$ for every $\sigma \in \varkappa^{<\omega}$. Then define another map $\xi: \varkappa^{<\omega} \rightarrow C(M, \mathbb{I})$ by setting $\xi[\emptyset]=\eta[\emptyset] / \eta[\emptyset]$ and, for every $\sigma \in \varkappa^{<\omega}$ and $\alpha<\varkappa$,

$$
\xi\left[\sigma^{\wedge} \alpha\right]= \begin{cases}\frac{\xi[\sigma] \cdot \eta\left[\sigma^{\wedge} \alpha\right]}{\sum\left\{\eta\left[\sigma^{\wedge} \delta\right]: \delta<\varkappa\right\}} & \text { provided } \mathcal{L}(\sigma) \neq \emptyset \\ \eta\left[\sigma^{\wedge} \alpha\right] & \text { otherwise. }\end{cases}
$$

This $\xi$ satisfies all our requirements.

Proof of Theorem 5.1. Let $(Y,\|\cdot\|)$ be a Banach space with $w(Y) \leq \lambda$, and let $f: A \times M \rightarrow Y$ be a continuous map. We now show that $f$ has a continuous extension $g: X \times M \rightarrow Y$.

For this purpose, with every space $T$ we associate an exponential correspondence $\Delta_{T}: C(T \times M, Y) \rightarrow C(T, Y)^{\varkappa^{<\omega}}$ defined in the following way. Let $\mathcal{S}: \varkappa^{<\omega} \rightarrow \mathcal{T}(M)$ be a strong sieve on $M$ which exists by Lemma 5.5. Pick a fixed dense subset $D$ of $M$ with $|D|=\varkappa$, and then define a map $\theta: \varkappa^{<\omega} \rightarrow M$ by $\theta(\sigma) \in D \cap \mathcal{S}(\sigma)$ for every $\sigma \in \varkappa^{<\omega}$. Finally, $\Delta_{T}$ is defined by $\Delta_{T}(h)[\sigma](x)=h(x, \theta(\sigma))$ for every $h \in C(T \times M, Y), \sigma \in \varkappa^{<\omega}$ and $x \in T$.

Now, let $\Phi=\Delta_{A}(f): \varkappa^{<\omega} \rightarrow C(A, Y)$. Since the correspondence $\Delta_{T}$ is injective, the statement that $f$ has a continuous extension $g$ to the whole of $X \times M$ becomes equivalent to the existence of a map $\Gamma: \varkappa^{<\omega} \rightarrow C(X, Y)$ 
subject to the extension condition

$$
\Gamma[\sigma] \mid A=\Phi[\sigma] \quad \text { for every } \sigma \in \varkappa^{<\omega},
$$

and, in addition, to the continuity condition

$$
\Gamma \in \Delta_{X}(C(X \times M, Y)) .
$$

Namely, a map $g \in C(X \times M, Y)$ is an extension of $f$ if and only if the corresponding map $\Gamma=\Delta_{X}(g)$ satisfies both (EC) and (CC). It is now clear that the hypothesis on $A$ to be $P^{\lambda}$-embedded in $X$ implies "many" solutions of (EC). In contrast, (CC) has no visible relationship with our hypotheses. In this regard, a key step in the proof is given by the following equivalent setting of $(\mathrm{CC})$. Let $\xi: \varkappa^{<\omega} \rightarrow C(M, \mathbb{I})$ be a sieve-partition of unity on $M$ as in Lemma 5.6 applied to the sieve $\mathcal{S}$. Then, as we demonstrate at the end of this proof, $h \in C(X \times M, Y)$ implies

$$
h=\lim _{n \rightarrow \infty} \sum\left\{\xi[\sigma] \cdot \Delta_{X}(h)[\sigma]: \sigma \in \varkappa^{n}\right\} .
$$

In view of this, $(\mathrm{CC})$ becomes now equivalent to the condition on $\Gamma$ that

$$
\lim _{n \rightarrow \infty} \sum\left\{\xi[\sigma] \cdot \Gamma[\sigma]: \sigma \in \varkappa^{n}\right\} \in C(X \times M, Y) .
$$

Having $(\mathrm{CC})^{\dagger}$ in mind, the final separating of the factors of the product $X \times M$ will be achieved by a condition on $\Gamma$ that concerns only $X$ and involves $\mathcal{S}$-free expansions of a sequence of decreasing $\mathcal{S}$-free maps generated by $\Phi$.

Turning to this, for every $\sigma \in \varkappa^{<\omega}$ we pick a fixed map $\mathcal{D}_{\sigma}: \varkappa \rightarrow D^{2}$ such that

$$
\mathcal{D}_{\sigma}(\varkappa)=(D \cap \mathcal{S}(\sigma))^{2} .
$$

Next, for every $k<\omega$ we define a map $\mathcal{G}_{k}: \varkappa^{<\omega} \rightarrow \mathcal{T}_{0}(A)^{\varkappa}$ by letting, for $\sigma \in \varkappa^{<\omega}$ and $\alpha<\varkappa$,

$$
\mathcal{G}_{k}[\sigma](\alpha)=\left\{x \in A: \operatorname{diam}\left(f\left(\{x\} \times \mathcal{D}_{\sigma}[\alpha](2)\right)\right)>1 / 2^{k+1}\right\} .
$$

Note that, by (5.4), $\beta<\varkappa$ implies $\mathcal{D}_{\sigma^{\wedge} \beta}[\varkappa] \subset \mathcal{D}_{\sigma}[\varkappa]$. Therefore $\mathcal{G}_{k}\left[\sigma^{\wedge} \beta\right](\varkappa)$ always refines $\mathcal{G}_{k}[\sigma](\varkappa)$. That is, each $\mathcal{G}_{k}, k<\omega$, is a decreasing map. Finally, observe also that, by (5.4) and (5.5), $\sigma \in \varkappa^{<\omega}$ implies

$$
\left\langle\mathcal{G}_{k}, \varkappa\right\rangle(\sigma)=\left\{x \in A: \operatorname{diam}(f(\{x\} \times \mathcal{S}(\sigma)))>1 / 2^{k+1}\right\} .
$$

Since our sieve $\mathcal{S}$ is strong and $f$ is continuous, by (5.6), each $\mathcal{G}_{k}, k<\omega$, is an $\mathcal{S}$-free map. Then each $\mathcal{G}_{k}$ has a decreasing $\mathcal{S}$-free expansion $\mathcal{H}_{k}$ : $\varkappa^{<\omega} \rightarrow \mathcal{T}_{0}(X)^{\varkappa}$. Indeed, by hypothesis, $\mathcal{G}_{k}$ has an $\mathcal{S}$-free expansion $\mathcal{H}_{k}^{\prime}$ : $\varkappa^{<\omega} \rightarrow \mathcal{T}_{0}(X)^{\varkappa}$. Then for every $\sigma \in \varkappa^{<\omega}$ and $\alpha, \tau<\varkappa$ just set $\mathcal{H}_{k}[\emptyset](\alpha)=$ $\mathcal{H}_{k}^{\prime}[\emptyset](\alpha)$ and $\mathcal{H}_{k}\left[\sigma^{\wedge} \tau\right](\alpha)=\mathcal{H}_{k}^{\prime}\left[\sigma^{\wedge} \tau\right](\alpha) \cap \mathcal{H}_{k}[\sigma](\beta)$, where $\beta<\varkappa$ is such that $\mathcal{G}_{k}\left[\sigma^{\wedge} \tau\right](\alpha) \subset \mathcal{G}_{k}[\sigma](\beta)$. 
The maps $\mathcal{H}_{k}, k<\omega$, will play an important role in our proof. So, we summarize some of their properties. For convenience, whenever $k<\omega$, we define maps $\mathcal{F}_{k}: \varkappa^{<\omega} \rightarrow \mathcal{C}(X)$ and $\mathcal{Z}_{k}: \varkappa^{<\omega} \rightarrow \mathcal{C}_{0}(X)^{\varkappa}$ by setting $\mathcal{F}_{k}(\sigma)=X \backslash\left\langle\mathcal{H}_{k}, \varkappa\right\rangle(\sigma)$ and $\mathcal{Z}_{k}[\sigma](\alpha)=X \backslash \mathcal{H}_{k}[\sigma](\alpha)$ for $\sigma \in \varkappa^{<\omega}$ and $\alpha<\varkappa$. Note that

$$
\mathcal{F}_{k}(\sigma)=\bigcap\left\{\mathcal{Z}_{k}[\sigma](\alpha): \alpha<\varkappa\right\} \quad \text { for every } \sigma \in \varkappa^{<\omega} .
$$

Since $\mathcal{H}_{k}$ is an expansion of $\mathcal{G}_{k}$, it follows from (5.5) and (5.6) that, for every $\sigma \in \varkappa^{<\omega}$ and $\alpha<\varkappa$,

$$
\operatorname{diam}\left(f\left(\{x\} \times \mathcal{D}_{\sigma}[\alpha](2)\right)\right) \leq 1 / 2^{k+1} \quad \text { for every } x \in \mathcal{Z}_{k}[\sigma](\alpha) \cap A,
$$

and

$$
\operatorname{diam}(f(\{x\} \times \mathcal{S}(\sigma))) \leq 1 / 2^{k+1} \quad \text { for every } x \in \mathcal{F}_{k}(\sigma) \cap A .
$$

We also need the following properties of $\mathcal{F}_{k}, k<\omega$, which are not directly related to the map $f$. Namely, since each $\mathcal{H}_{k}$ is decreasing, the following holds for every $t \in \varkappa^{\omega}$ :

$$
\mathcal{F}_{k}(t \mid m) \subset \mathcal{F}_{k}(t \mid n) \quad \text { whenever } m \leq n<\omega .
$$

Since $\mathcal{H}_{k}$ is $\mathcal{S}$-free, $\bigcap\left\{\operatorname{cl}_{X}\left(\left\langle\mathcal{H}_{k}, \varkappa\right\rangle(t \mid n)\right): n<\omega\right\}=\emptyset$ for every $t \in \Sigma_{\omega}(z)$ and every $z \in M$. Hence, by Lemma 5.2 , for every point $z \in M$ we have

$$
X=\bigcup\left\{\bigcap\left\{\operatorname{int}_{X}\left(\mathcal{F}_{k}(\sigma)\right): \sigma \in \Sigma_{n}(z)\right\}: n<\omega\right\} .
$$

Here, $\operatorname{int}_{X}(Z)$ means the interior of $Z$ in $X$. To these properties, we add another one related to the map $\Phi=\Delta_{A}(f): \varkappa^{<\omega} \rightarrow C(A, Y)$ which is sufficient to restore in full the former map $f$. Namely, according to the definition of $\Phi,(5.9)$ and (5.10), we see that, for every $k<\omega, m \leq n<\omega$ and $\sigma \in \varkappa^{n}$,

(‡) $\quad\|\Phi[\sigma](x)-\Phi[\sigma \mid m](x)\| \leq 1 / 2^{k+1} \quad$ whenever $x \in \mathcal{F}_{k}(\sigma \mid m) \cap A$.

We are now ready to construct the promised $\Gamma: \varkappa^{<\omega} \rightarrow C(X, Y)$ satisfying both (EC) and (CC). In view of $(\mathrm{CC})^{\dagger}$, our final reduction of $(\mathrm{CC})$ is given by the requirement on $\Gamma$ that, for every $k \leq m \leq n<\omega$ and $\sigma \in \varkappa^{n}$,

$(\mathrm{CC})^{\ddagger} \quad\|\Gamma[\sigma](x)-\Gamma[\sigma \mid m](x)\| \leq 1 / 2^{k} \quad$ whenever $x \in \mathcal{F}_{k}(\sigma \mid m)$.

Construction of $\Gamma$. By induction on $n<\omega$, we shall construct a sequence of maps $\Gamma_{n}: \varkappa^{n} \rightarrow C(X, Y)$ such that, for every $k \leq m \leq n<\omega$ and $\sigma \in \varkappa^{n}$,

$$
\Gamma_{n}[\sigma] \mid A=\Phi[\sigma]
$$

and

$$
\left\|\Gamma_{n}[\sigma](x)-\Gamma_{m}[\sigma \mid m](x)\right\| \leq 1 / 2^{k} \quad \text { whenever } x \in \mathcal{F}_{k}(\sigma \mid m) .
$$


If this is done, then we may define $\Gamma: \varkappa^{<\omega} \rightarrow C(X, Y)$ by $\Gamma \mid \varkappa^{n}=$ $\Gamma_{n}$ for every $n<\omega$. According to (5.12) and (5.13), it will satisfy all our requirements.

Thus, it only remains to define these $\Gamma_{n}$ 's. Since $A$ is $P^{\lambda}$-embedded in $X$, by definition, there is a map $\Omega: \varkappa^{<\omega} \rightarrow C(X, Y)$ satisfying (EC), i.e.

$$
\Omega[\sigma] \mid A=\Phi[\sigma] \quad \text { for every } \sigma \in \varkappa^{<\omega} .
$$

Then, setting $\Gamma_{0}=\Omega \mid \varkappa^{0}$, we may suppose that, for some $n<\omega$, the maps $\Gamma_{m}: \varkappa^{m} \rightarrow C(X, Y), m \leq n$, have already been defined so that (5.12) and (5.13) hold, and let us now demonstrate how $\Gamma_{n+1}: \varkappa^{n+1} \rightarrow C(X, Y)$ can be constructed. To prepare for this, we need the following observation.

Claim 1. For every $\sigma \in \varkappa^{n+1}$ and $k \leq m \leq n$, there exists a zero-set $B_{k m}(\sigma)$ of $X$ such that $B_{k m}(\sigma) \cap A=\emptyset$ and

$$
\left\|\Omega[\sigma](x)-\Gamma_{m}[\sigma \mid m](x)\right\|<1 / 2^{k} \quad \text { for every } x \in \mathcal{F}_{k}(\sigma \mid m) \backslash B_{k m}(\sigma) .
$$

Proof. We consider the set $O=\left\{x \in X:\left\|\Omega[\sigma](x)-\Gamma_{m}[\sigma \mid m](x)\right\|<\right.$ $\left.1 / 2^{k}\right\}$. Note that, by (5.14) and (5.12), we have $\Omega[\sigma](x)=\Phi[\sigma](x)=$ $f(x, \theta(\sigma))$ and $\Gamma_{m}[\sigma \mid m](x)=\Phi[\sigma \mid m](x)=f(x, \theta(\sigma \mid m))$ for every $x \in A$. On the other hand, $\theta(\sigma) \in D \cap \mathcal{S}(\sigma) \subset D \cap \mathcal{S}(\sigma \mid m)$ and $\theta(\sigma \mid m) \in D \cap \mathcal{S}(\sigma \mid m)$. Hence, by (5.4), there exists $\beta<\varkappa$ such that $\mathcal{D}_{\sigma \mid m}[\beta](2)=\{\theta(\sigma), \theta(\sigma \mid m)\}$. For this particular $\beta$, by (5.7) and (5.8), we finally get

$$
\mathcal{F}_{k}(\sigma \mid m) \cap A \subset \mathcal{Z}_{k}[\sigma \mid m](\beta) \cap A \subset O .
$$

Then $B_{k m}(\sigma)=\mathcal{Z}_{k}[\sigma \mid m](\beta) \backslash O$ is as required.

Whenever $\sigma \in \varkappa^{n+1}$, let $B_{k m}(\sigma), k \leq m \leq n$, be as in Claim 1. Then $B(\sigma)=\bigcup\left\{B_{k m}(\sigma): k \leq m \leq n\right\}$ is a zero-set of $X$ as a finite union of zero-sets of $X$ and, by Claim $1, A \cap B(\sigma)=\emptyset$. Since $A$ is $C$-embedded in $X$, by Lemma 3.1, there is $h_{\sigma} \in C(X, \mathbb{I})$ such that $A \subset h_{\sigma}^{-1}(1)$ and $B(\sigma) \subset h_{\sigma}^{-1}(0)$. Now, define $\Gamma_{n+1}[\sigma] \in C(X, Y)$ by letting, for every $x \in X$,

$$
\Gamma_{n+1}[\sigma](x)=\left(1-h_{\sigma}(x)\right) \cdot \Gamma_{n}[\sigma \mid n](x)+h_{\sigma}(x) \cdot \Omega[\sigma](x) .
$$

In this way, we get a map $\Gamma_{n+1}: \varkappa^{n+1} \rightarrow C(X, Y)$. To show that it is as required, take $\sigma \in \varkappa^{n+1}$. Then, by (5.14), $\Gamma_{n+1}[\sigma]|A=\Omega[\sigma]| A=\Phi[\sigma]$ because $A \subset h_{\sigma}^{-1}(1)$. That is, (5.12) holds. In order to check (5.13), let $k \leq m<n+1$ and $x \in \mathcal{F}_{k}(\sigma \mid m)$. Since $\Gamma_{n}$ and $\Gamma_{m}$ are as in (5.13), we have

$$
\left\|\Gamma_{n}[\sigma \mid n](x)-\Gamma_{m}[\sigma \mid m](x)\right\|<1 / 2^{k} .
$$

If $x \in B(\sigma)$, then $\Gamma_{n+1}[\sigma](x)=\Gamma_{n}[\sigma \mid n](x)$ because $B(\sigma) \subset h_{\sigma}^{-1}(0)$. Therefore, by (5.15), $\left\|\Gamma_{n+1}[\sigma](x)-\Gamma_{m}[\sigma \mid m](x)\right\|<1 / 2^{k}$. In case $x \notin B(\sigma)$, it follows from Claim 1 that $\left\|\Omega[\sigma](x)-\Gamma_{m}[\sigma \mid m](x)\right\|<1 / 2^{k}$. This, together with (5.15), finally implies that 


$$
\begin{aligned}
\left\|\Gamma_{n+1}[\sigma](x)-\Gamma_{m}[\sigma \mid m](x)\right\|= & \|\left(1-h_{\sigma}(x)\right) \cdot\left(\Gamma_{n}[\sigma \mid n](x)-\Gamma_{m}[\sigma \mid m](x)\right) \\
& +h_{\sigma}(x) \cdot\left(\Omega[\sigma](x)-\Gamma_{m}[\sigma \mid m](x)\right) \| \\
\leq & \left(1-h_{\sigma}(x)\right) \cdot\left\|\Gamma_{n}[\sigma \mid n](x)-\Gamma_{m}[\sigma \mid m](x)\right\| \\
& +h_{\sigma}(x) \cdot\left\|\Omega[\sigma](x)-\Gamma_{m}[\sigma \mid m](x)\right\| \\
< & \left(1-h_{\sigma}(x)\right) \cdot 1 / 2^{k}+h_{\sigma}(x) \cdot 1 / 2^{k}=1 / 2^{k} .
\end{aligned}
$$

Thus, (5.13) holds as well.

Construction of $g \in C(X \times M, Y)$ with $\Delta_{X}(g)=\Gamma$. Let $\xi: \varkappa^{<\omega} \rightarrow$ $C(M, \mathbb{I})$ be as in Lemma 5.6. Note that, for every $m \leq n<\omega, \tau \in \varkappa^{m}$ and $z \in M$,

$$
\xi[\tau](z)=\sum\left\{\xi[\sigma](z): \sigma \in \varkappa^{n} \text { and } \sigma \mid m=\tau\right\} .
$$

Then, for each $n<\omega$, we define a map $g_{n}: X \times M \rightarrow Y$ by setting, for $(x, z) \in X \times M$,

$$
g_{n}(x, z)=\sum\left\{\xi[\sigma](z) \cdot \Gamma[\sigma](x): \sigma \in \varkappa^{n}\right\} .
$$

According to Lemma 5.6, the above definition is correct because $Y$ is a Banach space and each family $\left\{\mathcal{S}(\sigma): \sigma \in \varkappa^{n}\right\}, n<\omega$, is locally finite. Moreover, $g_{n}$ is a continuous map. In order to check this, for every $n<\omega$ and $z \in M$, let $\mathcal{L}_{n}(z)$ be as in (5.2). Then $\mathcal{L}_{n}(z)$ is a neighbourhood of $z$ in $M$ such that, by (5.3), $\Sigma_{n}(y) \subset \Sigma_{n}(z)$ for every $y \in \mathcal{L}_{n}(z)$. On the other hand, by Lemma 5.6 ,

$$
\xi[\sigma](y)=0 \quad \text { for every } \sigma \in \varkappa^{n} \backslash \Sigma_{n}(y) .
$$

This finally implies that

$$
g_{n}(x, y)=\sum\left\{\xi[\sigma](y) \cdot \Gamma[\sigma](x): \sigma \in \Sigma_{n}(z)\right\} \quad \text { for }(x, y) \in X \times \mathcal{L}_{n}(z) .
$$

Hence, $g_{n}$ is continuous because $\Sigma_{n}(z)$ is finite.

The following two claims collect a bit more information about the maps $g_{n}$.

Claim 2. Whenever $(x, z) \in X \times M$ and $m \leq n<\omega$, we have

$$
g_{m}(x, z)=\sum\left\{\xi[\sigma](z) \cdot \Gamma[\sigma \mid m](x): \sigma \in \Sigma_{n}(z)\right\} .
$$

Proof. It follows from (5.16) and (5.17) that

$$
\begin{aligned}
g_{m}(x, z) & =\sum\left\{\xi[\tau](z) \cdot \Gamma[\tau](x): \tau \in \Sigma_{m}(z)\right\} \\
& =\sum\left\{\sum\left\{\xi[\sigma](z): \sigma \in \varkappa^{n} \text { and } \sigma \mid m=\tau\right\} \cdot \Gamma[\tau](x): \tau \in \Sigma_{m}(z)\right\} \\
& =\sum\left\{\xi[\sigma](z) \cdot \Gamma[\sigma \mid m](x): \sigma \in \varkappa^{n} \text { and } \sigma \mid m \in \Sigma_{m}(z)\right\} .
\end{aligned}
$$

Since $\Sigma_{n}(z) \subset\left\{\sigma \in \varkappa^{n}: \sigma \mid m \in \Sigma_{m}(z)\right\}$, (5.17) completes the proof. 
Claim 3. For every $(x, z) \in X \times M$ and $k<\omega$, there exists a neighbourhood $U$ of $(x, z)$ in $X \times M$ and an $m<\omega$ such that

$$
\left\|g_{n}(y, s)-g_{m}(y, s)\right\|<1 / 2^{k} \quad \text { whenever }(y, s) \in U \text { and } m \leq n<\omega .
$$

Proof. By (5.11), there exists $m<\omega$ such that

$$
x \in V=\bigcap\left\{\operatorname{int}_{X}\left(\mathcal{F}_{k+1}(\sigma)\right): \sigma \in \Sigma_{m}(z)\right\} .
$$

Let us show that $U=V \times \mathcal{L}_{m}(z)$ satisfies all our requirements, where $\mathcal{L}_{m}(z)$ is as in (5.2). To this end, take a point $(y, s) \in U$. Then, by (5.3), $\Sigma_{m}(s) \subset \Sigma_{m}(z)$, and therefore $y \in \bigcap\left\{\mathcal{F}_{k+1}(\tau): \tau \in \Sigma_{m}(s)\right\}$. Hence, by $(\mathrm{CC})^{\ddagger}, n \geq m$ implies

$$
\|\Gamma[\sigma](y)-\Gamma[\sigma \mid m](y)\|<1 / 2^{k+1} \quad \text { for every } \sigma \in \Sigma_{n}(s)
$$

because $\Sigma_{n}(s) \subset\left\{\sigma \in \varkappa^{n}: \sigma \mid m \in \Sigma_{m}(s)\right\}$. Then, by (5.17) and Claim 2, this finally implies that, for every $n \geq m$,

$$
\begin{aligned}
\left\|g_{n}(y, s)-g_{m}(y, s)\right\| & \leq \sum\left\{\xi[\sigma](s) \cdot\|\Gamma[\sigma](y)-\Gamma[\sigma \mid m](y)\|: \sigma \in \Sigma_{n}(s)\right\} \\
& \leq \sum\left\{\xi[\sigma](s) \cdot \frac{1}{2^{k+1}}: \sigma \in \Sigma_{n}(s)\right\} \\
& =\frac{1}{2^{k+1}} \sum\left\{\xi[\sigma](s): \sigma \in \varkappa^{n}\right\} \\
& =\frac{1}{2^{k+1}} \cdot \xi[\emptyset](s)=\frac{1}{2^{k+1}} . \text { - }
\end{aligned}
$$

For $(x, z) \in X \times M$, it follows from Claim 3 that $\left\{g_{n}(x, z): n<\omega\right\}$ is a Cauchy sequence in the Banach space $(Y,\|\cdot\|)$. So, it must converge to some point of $Y$. Hence, we can define a map $g: X \times M \rightarrow Y$ by

$$
g(x, z)=\lim _{n \rightarrow \infty} g_{n}(x, z) \quad \text { for every }(x, z) \in X \times M .
$$

This map $g$ is continuous. Indeed, take a point $(x, z) \in X \times M$ and an $\varepsilon>0$. According to Claim 3, there exists a neighbourhood $V$ of $(x, z) \in X \times M$ and an $m<\omega$ such that $n \geq m$ implies

$$
\left\|g_{n}(y, s)-g_{m}(y, s)\right\|<\varepsilon / 3 \quad \text { for every }(y, s) \in V .
$$

Hence, in particular, $\left\|g(y, s)-g_{m}(y, s)\right\| \leq \varepsilon / 3$ for every $(y, s) \in V$. Because of the continuity of $g_{m}$, there also exists a neighbourhood $W$ of $(x, z) \in$ $X \times M$ such that $\left\|g_{m}(x, z)-g_{m}(y, s)\right\|<\varepsilon / 3$ for every $(y, s) \in W$. Thus, whenever $(y, s) \in V \cap W$, we get

$$
\begin{aligned}
\|g(x, z)-g(y, s)\| \leq & \left\|g(x, z)-g_{m}(x, z)\right\| \\
& +\left\|g_{m}(x, z)-g_{m}(y, s)\right\|+\left\|g_{m}(y, s)-g(y, s)\right\| \\
< & \varepsilon / 3+\varepsilon / 3+\varepsilon / 3=\varepsilon,
\end{aligned}
$$

which certainly implies that $g$ is continuous. 
We complete the proof of Theorem 5.1 by showing that $g$ is an extension of $f$. Let $(x, z) \in A \times M$, and let $\varepsilon>0$. Since our sieve $\mathcal{S}$ is strong, the collection $\left\{\mathcal{S}_{n}(z): n<\omega\right\}$ constitutes a local base at the point $z$ in $M$. Hence, there exists $m<\omega$ with $\|f(x, z)-f(x, y)\|<\varepsilon$ for every $y \in \mathcal{S}_{m}(z)$. On the other hand, by (EC), $\Gamma[\sigma](x)=\Phi[\sigma](x)=f(x, \theta(\sigma))$ for every $\sigma \in \varkappa^{<\omega}$. Since $\theta(\sigma) \in \mathcal{S}(\sigma) \subset \mathcal{S}_{n}(z) \subset \mathcal{S}_{m}(z)$ for every $\sigma \in \Sigma_{n}(z)$ and $n \geq m$, this implies that

$$
\|f(x, z)-\Gamma[\sigma](x)\|<\varepsilon \quad \text { whenever } \sigma \in \Sigma_{n}(z) \text { and } n \geq m .
$$

In addition, by (5.17), we have

$$
f(x, z)=\sum\left\{\xi[\sigma](z) \cdot f(x, z): \sigma \in \Sigma_{n}(z)\right\} \quad \text { for every } n<\omega .
$$

This finally implies that, for every $n \geq m$,

$$
\begin{aligned}
\left\|f(x, z)-g_{n}(x, z)\right\| & \leq \sum\left\{\xi[\sigma](z) \cdot\|f(x, z)-\Gamma[\sigma](x)\|: \sigma \in \Sigma_{n}(z)\right\} \\
& <\sum\left\{\xi[\sigma](z) \cdot \varepsilon: \sigma \in \Sigma_{n}(z)\right\} \\
& =\varepsilon \cdot \sum\left\{\xi[\sigma](z): \sigma \in \varkappa^{n}\right\}=\varepsilon \cdot \xi[\emptyset](z)=\varepsilon .
\end{aligned}
$$

That is, $f(x, z)=\lim _{n \rightarrow \infty} g_{n}(x, z)=g(x, z)$.

6. Proof of Theorem 1.1. According to Proposition 3.6 and Theorems 4.1 and 5.1, the following two propositions finally accomplish the proof of Theorem 1.1.

Proposition 6.1. Let $A$ be a subset of a topological space $X$, and let $M$ be a weight-homogeneous nowhere locally compact metric space such that $A \times M$ is $C^{*}$-embedded in $X \times M$. Let $\varkappa=w(M)$ and let $\mathcal{S}: \varkappa^{<\omega} \rightarrow \mathcal{T}(M)$ be a strong sieve on $M$. Then every decreasing $\mathcal{S}$-free map $\mathcal{G}: \varkappa^{<\omega} \rightarrow \mathcal{T}_{0}(A)^{\varkappa}$ admits an $\mathcal{S}$-free expansion $\mathcal{H}: \varkappa^{<\omega} \rightarrow \mathcal{T}_{0}(X)^{\varkappa}$.

Pr o of. Let $\mathcal{G}: \varkappa^{<\omega} \rightarrow \mathcal{T}_{0}(A)^{\varkappa}$ be a decreasing $\mathcal{S}$-free map. Since each cozero-set is a countable union of zero-sets, for every $\sigma \in \varkappa^{<\omega}$ there exists a map $\mathcal{Z}_{\sigma}: \varkappa \rightarrow \mathcal{C}_{0}(A)^{\omega}$ such that $\mathcal{G}[\sigma](\alpha)=\left\langle\mathcal{Z}_{\sigma}, \omega\right\rangle(\alpha)$ for every $\alpha<\varkappa$. On the other hand, by [27, Lemma], there is a map $p: \varkappa^{<\omega} \rightarrow M^{2}$ such that, for every $\sigma \in \varkappa^{<\omega}$ and $\tau \in \varkappa^{<\omega} \backslash\{\sigma\}$,

$$
p[\sigma](0) \neq p[\sigma](1) \quad \text { and } \quad p[\sigma](2) \subset \mathcal{S}(\sigma) \backslash p[\tau](2) .
$$

For every $\sigma \in \varkappa^{<\omega}$ and $i<2$, we let

$$
Z_{i}(\sigma)=\bigcup\left\{\mathcal{Z}_{\sigma}[\alpha](n) \times\left\{p\left[\sigma^{\wedge} \hat{\alpha} n\right](i)\right\}: \alpha<\varkappa \text { and } n<\omega\right\} .
$$

Note that $Z_{0}(\sigma) \cup Z_{1}(\sigma) \subset A \times \mathcal{S}\left(\sigma^{\wedge} \alpha^{\wedge} n\right)$ for each $\alpha<\varkappa$ and $n<\omega$, while $\left\{A \times \mathcal{S}\left(\sigma^{\wedge} \alpha^{\hat{n}} n\right): \alpha<\varkappa\right.$ and $\left.n<\omega\right\}$ is a locally finite family of cozero-sets of $A \times M$. Hence, by Lemma 2.4, both $Z_{0}(\sigma)$ and $Z_{1}(\sigma)$ are zero-sets of $A \times M$. 
Whenever $\sigma \in \varkappa^{<\omega}$, we now set $W_{\sigma}=\bigcup\left\{\mathcal{G}[\sigma](\alpha) \times \mathcal{S}\left(\sigma^{\wedge} \alpha\right): \alpha<\varkappa\right\}$ which defines a cozero-set of $A \times M$ such that

$$
Z_{0}(\sigma) \cup Z_{1}(\sigma) \subset W_{\sigma} \subset\langle\mathcal{G}, \varkappa\rangle(\sigma) \times \mathcal{S}(\sigma) .
$$

Then, by Lemma 5.3, $\left\{W_{\sigma}: \sigma \in \varkappa^{<\omega}\right\}$ is a locally finite family of cozero-sets of $A \times M$. Relying once again on Lemma 2.4, we find that $Z_{i}=\bigcup\left\{Z_{i}(\sigma)\right.$ : $\left.\sigma \in \varkappa^{<\omega}\right\}$ is a zero-set of $A \times M$ for every $i<2$. Moreover, by (6.1), $Z_{0} \cap Z_{1}=\emptyset$. Since $A \times M$ is $C^{*}$-embedded in $X \times M$, this implies the existence of $g \in C(X \times M)$ such that $Z_{i} \subset g^{-1}(i), i<2$. For every $\sigma \in \varkappa^{<\omega}$ and $\alpha<\varkappa$, we now let

$$
\mathcal{H}[\sigma](\alpha)=\bigcup\left\{\left\{x \in X: \operatorname{diam}\left(g\left(\{x\} \times p\left[\sigma^{\wedge} \alpha^{\wedge} n\right](2)\right)\right)>2 / 3\right\}: n<\omega\right\} .
$$

Thus, we get a map $\mathcal{H}: \varkappa^{<\omega} \rightarrow \mathcal{T}_{0}(X)^{\varkappa}$ which is an expansion of $\mathcal{G}$. Indeed, $x \in \mathcal{G}[\sigma](\alpha)$ implies that $x \in \mathcal{Z}_{\sigma}[\alpha](n)$ for some $n<\omega$. Therefore, $x \in$ $\mathcal{H}[\sigma](\alpha)$ because $\operatorname{diam}\left(g\left(\{x\} \times p\left[\sigma^{\wedge} \alpha^{\wedge} n\right](2)\right)\right)=1$.

It only remains to show that $\mathcal{H}$ is $\mathcal{S}$-free. Take $t \in \Sigma_{\omega}(z)$ for some $z \in M$. Also, let $x \in X$. Since $g$ is continuous at $(x, z) \in X \times M$ and $\mathcal{S}$ is a strong sieve, there is a neighbourhood $U$ of $x$ and $m<\omega$ such that

$$
\operatorname{diam}(g(U \times \mathcal{S}(t \mid m)))<2 / 3 .
$$

By (6.1), we have $p\left[(t \mid m)^{\wedge} \alpha^{\wedge} n\right](2) \subset \mathcal{S}\left((t \mid m)^{\wedge} \alpha^{\wedge} n\right) \subset \mathcal{S}(t \mid m)$ for all $\alpha<\varkappa$ and $n<\omega$. According to the definition of $\mathcal{H}[t \mid m](\sigma)$ and (6.2), this finally implies that $U \cap \mathcal{H}[t \mid m](\alpha)=\emptyset$ for all $\alpha<\varkappa$. That is, $x \notin \mathrm{cl}_{X}(\langle\mathcal{H}, \varkappa\rangle(t \mid m))$, which completes the proof.

Proposition 6.2. Let $A$ be a subset of a topological space $X$, and let $M$ be a weight-homogeneous nowhere locally compact metric space such that $A \times M$ is $U^{\omega}$-embedded in $X \times M$. Let $\varkappa=w(M)$ and let $\mathcal{S}: \varkappa^{<\omega} \rightarrow \mathcal{T}(M)$ be a strong sieve on $M$. Then every decreasing $\mathcal{S}$-free map $\mathcal{G}: \varkappa^{<\omega} \rightarrow \mathcal{T}_{0}(A)^{\varkappa}$ admits an $\mathcal{S}$-free expansion $\mathcal{H}: \varkappa^{<\omega} \rightarrow \mathcal{T}_{0}(X)^{\varkappa}$.

Pr o of. Let $\mathcal{G}: \varkappa^{<\omega} \rightarrow \mathcal{T}_{0}(A)^{\varkappa}$ be a decreasing $\mathcal{S}$-free map. As before, for every $\sigma \in \varkappa^{<\omega}$ there is a map $\mathcal{Z}_{\sigma}: \varkappa \rightarrow \mathcal{C}_{0}(A)^{\omega}$ such that $\mathcal{G}[\sigma](\alpha)=$ $\left\langle\mathcal{Z}_{\sigma}, \omega\right\rangle(\alpha)$ for every $\alpha<\varkappa$. Also, let $q: \varkappa^{<\omega} \rightarrow M$ be such that, for every $\sigma \in \varkappa^{<\omega}$ and $\tau \in \varkappa^{<\omega} \backslash\{\sigma\}$,

$$
q(\sigma) \in \mathcal{S}(\sigma) \backslash\{q(\tau)\} .
$$

Following the previous proof, for every $\sigma \in \varkappa^{<\omega}$, we now define a zero-set $Q(\sigma)=\bigcup\left\{\mathcal{Z}_{\sigma}[\alpha](n) \times\left\{q\left(\sigma^{\wedge} \alpha \hat{n}\right)\right\}: \alpha<\varkappa\right.$ and $\left.n<\omega\right\}$ of $A \times M$, and a cozero-set $W_{\sigma}=\bigcup\left\{\mathcal{G}[\sigma](\alpha) \times \mathcal{S}\left(\sigma^{\wedge} \alpha\right): \alpha<\varkappa\right\}$ of $A \times M$. In this way, for every $\sigma \in \varkappa^{<\omega}$ we have

$$
Q(\sigma) \subset W_{\sigma} \subset\langle\mathcal{G}, \varkappa\rangle(\sigma) \times \mathcal{S}(\sigma) .
$$


By Lemma 5.3, $\left\{W_{\sigma}: \sigma \in \varkappa^{<\omega}\right\}$ is locally finite in $A \times M$. According to Lemma 2.4, for every $k<\omega$ we can define a zero-set of $A \times M$ by letting

$$
F_{k}=\bigcup\left\{Q(\sigma): \sigma \in \varkappa^{<\omega} \backslash \varkappa^{<k}\right\} .
$$

Then $\left\{F_{k}: k<\omega\right\}$ is a decreasing sequence such that $\bigcap\left\{F_{k}: k<\omega\right\}=\emptyset$ because $\left\{Q(\sigma): \sigma \in \varkappa^{<\omega}\right\}$ is locally finite. Since $A \times M$ is $U^{\omega}$-embedded in $X \times M$, by Lemma 2.5 , we can find a decreasing sequence $\left\{Z_{k}: k<\omega\right\}$ of zero-sets of $X \times M$ such that $\bigcap\left\{Z_{k}: k<\omega\right\}=\emptyset$ and $F_{k} \subset Z_{k}, k<\omega$. Whenever $k<\omega$, take a continuous $g_{k}: X \times M \rightarrow\left[0,1 / 2^{k+1}\right]$ such that $Z_{k}=g_{k}^{-1}(0)$, and then let $g=\sum\left\{g_{k}: k<\omega\right\}$. Thus, we get $g \in C(X \times M)$ with the property that

$$
g(X \times M) \subset(0,1] \text { and } g\left(Z_{k}\right) \subset\left[0,1 / 2^{k}\right) \text { for every } k<\omega .
$$

Whenever $\alpha<\varkappa$ and $\sigma \in \varkappa^{k}$ for some $k<\omega$, we may now set

$$
\mathcal{H}[\sigma](\alpha)=\bigcup\left\{\left\{x \in X: g\left(x, q\left(\sigma^{\wedge} \hat{\wedge} n\right)\right)<1 / 2^{k}\right\}: n<\omega\right\} .
$$

In fact, this defines a map $\mathcal{H}: \varkappa^{<\omega} \rightarrow \mathcal{T}_{0}(X)^{\varkappa}$ which is an expansion of $\mathcal{G}$. Indeed, $x \in \mathcal{G}[\sigma](\alpha)$ implies $x \in \mathcal{Z}_{\sigma}[\alpha](n)$ for some $n<\omega$. Therefore, $\left(x, q\left(\sigma^{\wedge} \alpha^{\hat{n}} n\right)\right) \in Q(\sigma) \subset F_{k} \subset Z_{k}$ because $\sigma \in \varkappa^{k}$. So, by (6.3) and (6.4), $x \in \mathcal{H}[\sigma](\alpha)$.

We finish the proof by showing that $\mathcal{H}$ is $\mathcal{S}$-free. Take $t \in \Sigma_{\omega}(z)$ for some $z \in M$. Also, let $x \in X$. Then, by (6.4), there exists $k<\omega$ such that $g(x, z) \in\left(1 / 2^{k}, 1\right]$. Since $g$ is continuous at $(x, z) \in X \times M$ and $\mathcal{S}$ is a strong sieve, there exists a neighbourhood $U$ of $x$ and an $m<\omega$, with $k \leq m$, such that

$$
g(U \times \mathcal{S}(t \mid m)) \subset\left(1 / 2^{k}, 1\right] .
$$

By $(6.3), q((t \mid m) \wedge \hat{n}) \in \mathcal{S}((t \mid m) \wedge \hat{\wedge} n) \subset \mathcal{S}(t \mid m)$ for every $\alpha<\varkappa$ and $n<\omega$. Hence, by (6.5), $U \cap \mathcal{H}[t \mid m](\alpha)=\emptyset$ for every $\alpha<\varkappa$ because $k \leq m$. That is, $x \notin \operatorname{cl}_{X}(\langle\mathcal{H}, \varkappa\rangle(t \mid m))$.

7. A solution to another problem of Przymusiński. The technique developed in the paper allows one to obtain also the following solution of [23, Problem 1].

TheOREm 7.1. For a subset $A$ of a topological space $X$ and an infinite cardinal $\varkappa$, the following conditions are equivalent:

(a) The set $A \times M$ is $C^{*}$-embedded in $X \times M$ for every metric space $M$ with $w(M) \leq \varkappa$.

(b) The set $A$ is $C$-embedded in $X$ and every decreasing map $\mathcal{G}: \varkappa^{<\omega} \rightarrow$ $\mathcal{T}_{0}(A)^{\varkappa}$ has an expansion $\mathcal{H}: \varkappa^{<\omega} \rightarrow \mathcal{T}_{0}(X)^{\varkappa}$ such that $\bigcap\left\{\operatorname{cl}_{X}(\langle\mathcal{H}, \varkappa\rangle(t \mid n))\right.$ : $n<\omega\}=\emptyset$ for every $t \in \varkappa^{\omega}$ with $\bigcap\left\{\operatorname{cl}_{A}(\langle\mathcal{G}, \varkappa\rangle(t \mid n)): n<\omega\right\}=\emptyset$. 
Pr o of. Suppose that $A$ is as in (a). Endowing $\varkappa$ with the discrete topology, we may identify $\varkappa^{\omega}$ with the universal zero-dimensional Baire space of weight $\varkappa$. Then $\varkappa^{\omega}$ is weight-homogeneous and nowhere locally compact. Define $\mathcal{S}: \varkappa^{<\omega} \rightarrow \mathcal{T}\left(\varkappa^{\omega}\right)$ by $\mathcal{S}(\sigma)=\left\{t \in \varkappa^{\omega}: t \mid n=\sigma\right\}$ for every $\sigma \in \varkappa^{n}$ and $n<\omega$. Note that $\mathcal{S}$ is a strong sieve on $\varkappa^{\omega}$, each $\mathcal{S}(\sigma)$ is a clopen subset of $\varkappa^{\omega}$, and

$$
t \in \bigcap\{\mathcal{S}(t \mid n): n<\omega\} \quad \text { for every } t \in \varkappa^{\omega} .
$$

We also need the set $T=\left\{t \in \varkappa^{\omega}: 0 \in t(\omega)\right\}$ which is both dense and open in $\varkappa^{\omega}$.

Let $\varkappa_{1}=\{\alpha+1: \alpha<\varkappa\}$. Next, for every $\alpha<\varkappa$, define $\varphi(\alpha)=\alpha+1$ and $\psi(\alpha+1)=\alpha$. In this way, we get two injections $\varphi: \varkappa \rightarrow \varkappa_{1}$ and $\psi: \varkappa_{1} \rightarrow \varkappa$ such that $\psi \circ \varphi=\mathrm{id}_{\varkappa}$, the identity map of $\varkappa$.

Suppose now that $\mathcal{G}: \varkappa^{<\omega} \rightarrow \mathcal{T}_{0}(A)^{\varkappa}$ is a decreasing map. With this map $\mathcal{G}$ we associate another one $\mathcal{G}_{1}: \varkappa^{<\omega} \rightarrow \mathcal{T}_{0}(A)^{\varkappa}$ defined by letting, for every $\sigma \in \varkappa^{n}$ and $n<\omega, \mathcal{G}_{1}[\sigma]=\mathcal{G}[\psi \circ \sigma]$ if $\sigma \in \varkappa_{1}^{n}$ and $\mathcal{G}_{1}[\sigma](\alpha)=\emptyset, \alpha<\varkappa$, otherwise. In this way, we get a decreasing map $\mathcal{G}_{1}$ such that for every $t \in T$ there exists an $n<\omega$ with

$$
\left\langle\mathcal{G}_{1}, \varkappa\right\rangle(t \mid n)=\emptyset .
$$

Then we define a subset $M$ of $\varkappa^{\omega}$ by

$$
M=\left\{t \in \varkappa^{\omega}: \bigcap\left\{\operatorname{cl}_{A}\left(\left\langle\mathcal{G}_{1}, \varkappa\right\rangle(t \mid n)\right): n<\omega\right\}=\emptyset\right\} .
$$

According to (7.2), $T \subset M$, which implies that $M$ is weight-homogeneous and nowhere locally compact because $T$ is a dense open subset of $\varkappa^{\omega}$. Finally, define a strong sieve $\mathcal{S}_{1}: \varkappa^{<\omega} \rightarrow \mathcal{T}(M)$ on $M$ by $\mathcal{S}_{1}(\sigma)=\mathcal{S}(\sigma) \cap M$ for every $\sigma \in \varkappa^{<\omega}$. By (7.1) and (7.3), $\mathcal{G}_{1}$ is $\mathcal{S}_{1}$-free. Then, by (a) and Proposition 6.1, the map $\mathcal{G}_{1}$ has an $\mathcal{S}_{1}$-free expansion $\mathcal{H}_{1}: \varkappa^{<\omega} \rightarrow \mathcal{T}_{0}(X)^{\varkappa}$. In particular, $\mathcal{H}_{1}$ satisfies

$$
\mathcal{G}_{1}[\sigma](\alpha) \subset \mathcal{H}_{1}[\sigma](\alpha) \quad \text { for every } \sigma \in \varkappa^{<\omega} \text { and } \alpha<\varkappa,
$$

and, by (7.1) and the definition of $\mathcal{S}_{1}$,

$$
\bigcap\left\{\operatorname{cl}_{X}\left(\left\langle\mathcal{H}_{1}, \varkappa\right\rangle(t \mid n)\right): n<\omega\right\}=\emptyset \quad \text { for every } t \in M .
$$

Define another map $\mathcal{H}: \varkappa^{<\omega} \rightarrow \mathcal{T}_{0}(X)^{\varkappa}$ by $\mathcal{H}[\sigma]=\mathcal{H}_{1}[\varphi \circ \sigma]$ for every $\sigma \in \varkappa^{<\omega}$. This $\mathcal{H}$ is as required in (b). To show that $\mathcal{H}$ is an expansion of $\mathcal{G}$, take a $\sigma \in \varkappa^{n}$ for some $n<\omega$. Then, by (7.4), $\alpha<\varkappa$ implies

$$
\mathcal{H}[\sigma](\alpha)=\mathcal{H}_{1}[\varphi \circ \sigma](\alpha) \supset \mathcal{G}_{1}[\varphi \circ \sigma](\alpha)=\mathcal{G}[\psi \circ \varphi \circ \sigma](\alpha)=\mathcal{G}[\sigma](\alpha)
$$

because $\varphi \circ \sigma \in \varkappa_{1}^{n}$ and $\psi \circ \varphi=\mathrm{id}_{\varkappa}$.

Finally, assume that $\bigcap\left\{\mathrm{cl}_{A}(\langle\mathcal{G}, \varkappa\rangle(t \mid n)): n<\omega\right\}=\emptyset$ for some $t \in \varkappa^{\omega}$. Then $\varphi \circ t \in M$, because $\varphi \circ t \mid n \in \varkappa_{1}^{n}, n<\omega$, and 


$$
\begin{aligned}
\bigcap\left\{\mathrm{cl}_{A}\left(\left\langle\mathcal{G}_{1}, \varkappa\right\rangle(\varphi \circ t \mid n)\right): n<\omega\right\} & =\bigcap\left\{\mathrm{cl}_{A}(\langle\mathcal{G}, \varkappa\rangle(\psi \circ \varphi \circ t \mid n)): n<\omega\right\} \\
& =\bigcap\left\{\operatorname{cl}_{A}(\langle\mathcal{G}, \varkappa\rangle(t \mid n)): n<\omega\right\}=\emptyset .
\end{aligned}
$$

Hence, it follows from (7.5) that

$$
\bigcap\left\{\mathrm{cl}_{X}(\langle\mathcal{H}, \varkappa\rangle(t \mid n)): n<\omega\right\}=\bigcap\left\{\mathrm{cl}_{X}\left(\left\langle\mathcal{H}_{1}, \varkappa\right\rangle(\varphi \circ t \mid n)\right): n<\omega\right\}=\emptyset .
$$

That is, $\mathcal{H}$ is as in (b). Since, by Lemma 2.3, $A$ is $C$-embedded in $X$, this completes the verification of (b).

Suppose now that $A$ satisfies (b), and let $M$ be a metric space with $w(M) \leq \varkappa$. Consider the product space $M_{0}=M \times \varkappa^{\omega}$, where $\varkappa^{\omega}$ is the Baire metric space of weight $\varkappa$. Then $M_{0}$ is weight-homogeneous and nowhere locally compact. Hence, it follows from Proposition 3.6 and Theorems 4.1 and 5.1 that $A \times M_{0}$ is $C^{*}$-embedded in $X \times M_{0}$. If we consider $M$ as a closed subspace of $M_{0}$, then, by Lemma 2.1, $A \times M$ is $C^{*}$-embedded in $A \times M_{0}$. Hence, finally, $A \times M$ is $C^{*}$-embedded in $X \times M$.

\section{References}

[1] R. A. Aló and L. I. Sennott, Extending linear space-valued functions, Math. Ann. 191 (1971), 79-86.

[2] - - - Collectionwise normality and the extension of functions on product spaces, Fund. Math. 74 (1972), 231-243.

[3] R. Arens, Extension of coverings, of pseudometrics, and linear-space-valued mappings, Canad. J. Math. 5 (1953), 211-215.

[4] R. L. Blair and A. W. Hager, Extensions of zero-sets and of real-valued functions, Math. Z. 136 (1974), 41-52.

[5] J. Dugundji, An extension of Tietze's theorem, Pacific J. Math. 1 (1951), 353-367.

[6] T. E. Gantner, Extension of uniformly continuous pseudometrics, Trans. Amer. Math. Soc. 132 (1968), 147-157.

[7] G. Gruenhage, Generalized metric spaces, in: Handbook of Set-Theoretic Topology, K. Kunen and J. E. Vaughan (eds.), North-Holland, Amsterdam, 1984, 423501.

[8] T. Hoshina, Spaces with a property related to uniformly local finiteness, Tsukuba J. Math. 6 (1982), 51-62.

[9] -, Extensions of mappings II, in: Topics in General Topology, K. Morita and J. Nagata (eds.), North-Holland, Amsterdam, 1989, 41-80.

[10] - Extensions of mappings, in: Recent Progress in General Topology, M. Hušek and J. van Mill (eds.), North-Holland, Amsterdam, 1992, 405-416.

[11] T. Hoshina and K. Yamazaki, $C^{*}$-embedding and $C$-embedding on products with certain metric factor, Topology Appl. 82 (1998), 195-204.

[12] J. R. Isbell, Uniform Spaces, Amer. Math. Soc., Providence, RI, 1964.

[13] T. Ishii and H. Ohta, Generalizations of $C$-embedding and their applications, Math. Japon. 23 (1978), 349-368.

[14] F. Ishikawa, On countably paracompact spaces, Proc. Japan Acad. 31 (1955), 686-687. 
[15] M. Katětov, Extensions of locally finite coverings, Colloq. Math. 6 (1958), 145151.

[16] K. Kunen, Set Theory. An Introduction to Independence Proofs, Stud. Logic Found. Math. 102, North-Holland, Amsterdam, 1983.

[17] K. Morita, Paracompactness and product spaces, Fund. Math. 50 (1962), 223-236.

[18] -, Products of normal spaces with metric spaces, Math. Ann. 154 (1964), 365-382.

[19] - On generalizations of Borsuk's homotopy extension theorem, Fund. Math. 88 (1975), 1-6.

[20] K. Morita and T. Hoshina, P-embedding and product spaces, ibid. 93 (1976), $71-80$.

[21] H. O hta, Rectangular normality of products with a metric factor, RIMS Kôkyûroku 823 (1993), 106-117.

[22] T. C. Przymusiński, Collectionwise normality and extensions of continuous functions, Fund. Math. 98 (1978), 75-81.

[23] - Extending functions from products with a metric factor and absolutes, Pacific J. Math. 101 (1982), 463-475.

[24] - Notes on extendability of continuous functions from products with a metric factor, unpublished note, May 1983.

[25] -, A solution to a problem of E. Michael, Pacific J. Math. 114 (1984), 235-242.

[26] T. C. Przymusiński and M. Wage, Collectionwise normality and extensions of locally finite coverings, Fund. Math. 109 (1980), 175-187.

[27] M. E. Rudin and M. Starbird, Products with a metric factor, General Topology Appl. 5 (1975), 235-248.

[28] H. L. Shapiro, Extensions of pseudometrics, Canad. J. Math 18 (1966), 981-998.

[29] M. Starbird, Extending maps from products, in: Studies in Topology, N. H. Stavrakas and K. R. Allen (eds.), Academic Press, New York, 1975, 559-564.

[30] K. Yamazaki, $C^{*}$-embedding and $C$-embedding on product spaces, Tsukuba J. Math. 21 (1997), 515-527.

School of Mathematical and Statistical Sciences Faculty of Science

University of Natal

King George V Avenue

Durban 4041, South Africa

E-mail: gutev@sci.und.ac.za
Faculty of Education

Shizuoka University Ohya, Shizuoka, 422-8529 Japan E-mail: echohta@ipc.shizuoka.ac.jp

Received 27 April 1999; in revised form 8 December 1999 